\title{
Transfer function for vital infrasound pressures between the carotid artery and the tympanic membrane
}

\author{
Kenji Furihata ${ }^{a)}$ and Masato Yamashita \\ Department of Electrical and Electronic Engineering, Faculty of Engineering, Shinshu University, 4-17-1 \\ Wakasato, Nagano, 380-8533 Japan
}

(Received 24 May 2012; revised 2 December 2012; accepted 5 December 2012)

\begin{abstract}
While occupational injury is associated with numerous individual and work-related risk factors, including long working hours and short sleep duration, the complex mechanisms causing such injuries are not yet fully understood. The relationship between the infrasound pressures of the tympanic membrane [ear canal pressure (ECP)], detected using an earplug embedded with a low-frequency microphone, and the carotid artery [carotid artery pressure (CAP)], detected using a stethoscope fitted with the same microphone, can be quantitatively characterized using systems analysis. The transfer functions of 40 normal workers (19 to 57 years old) were characterized, involving the analysis of 446 data points. The ECP waveform exhibits a pulsatile character with a slow respiratory component, which is superimposed on a biphasic recording that is synchronous with the cardiac cycle. The respiratory ECP waveform correlates with the instantaneous heart rate. The results also revealed that various fatigue-related risk factors may affect the mean magnitudes of the measured pressures and the delay transfer functions between $\mathrm{CAP}$ and $\mathrm{ECP}$ in the study population; these factors include systolic blood pressure, salivary amylase activity, age, sleep duration, postural changes, chronic fatigue, and pulse rate. (C) 2013 Acoustical Society of America.
\end{abstract}

[http://dx.doi.org/10.1121/1.4773270]

PACS number(s): 43.80.Qf, 43.80.Vj [CCC]

Pages: $1169-1186$

\section{INTRODUCTION}

The objective of this paper is to characterize the relationship between the waveform associated with ear canal pressure (ECP) caused by vital infrasonic emissions from the tympanic membrane (TM) and the carotid artery pressure (CAP). This waveform has the same dimensions as the sound pressure and is detected using a stethoscope microphone. Specifically, the relationship between ECP and CAP can be characterized using the time-varying transfer function. The input signal for this technique is the CAP, which is composed of multiple frequencies, and the CAP is translated into an output ECP according to the transfer function imposed by the intracranial system on the input signal. The transfer function can change with time and with physiological and psychological factors. An experiment was designed to analyze time-varying behavior and the effects of other parameters (such as changes in posture, fatigue, and age) in healthy subjects. The experiment generated a non-trivial amount of data that was analyzed to generate ranges of "normal" values.

Intracranial pressure (ICP) is the pressure inside the skull that is exerted on the brain tissue, cerebrospinal fluid, and circulating blood volume in the brain. At rest, the ICP is normally between $5-15 \mathrm{mmHg}$, but this value fluctuates as it is affected by various factors including blood pulsation, respiration, postural changes, coughing, and exercise. Noninvasive monitoring of the ICP via the auditory system is theoretically possible because changes in ICP transfer to the

\footnotetext{
a) Author to whom correspondence should be addressed. Electronic mail: kennfur@shinshu-u.ac.jp
}

inner ear through connections between the cerebral spinal fluid and the cochlear fluids (Ciuman, 2009).

Matsuyama (1995) analyzed the external auditory canal pressures (EACPs) in adult cats using a contact-type pressure sensor. Simultaneously, measurements of arterial blood pressure (ABP) and ICP were taken from the cisterna magna. The typical EACP wave had one negative peak and several positive peaks. The EACP spectrum was composed of three main waves, exhibiting a pattern similar to that of the ICP pulse wave. The EACP is mainly derived from the ABP. During intracranial hypertension, the latency period between the first positive peak and the negative peak became shorter, and the pattern changed to a multi-peak shape. When ABP was elevated, the amplitude of EACP was also higher.

The inner ear is a bony labyrinth filled with perilymph, which contains a membranous labyrinth. The inner ear includes the cochlea and the semicircular canals of the vestibular system. The inner ear is connected to the subarachnoid space by the cochlear duct in the region of the posterior petrous bone. ICP is transmitted via the aforementioned structures to the TM and can be assessed based on infrasonic emissions (Marchbanks et al., 1987; Reid et al., 1989). Reid et al. (1990) found that patients with normal versus elevated ICPs showed differences in the degree of tympanic membrane displacement in response to the stimulation of the stapedial reflex. A non-invasive method of assessing ICP would be beneficial for patients with any abnormal cerebral pathology that increases ICP. In particular, such a method would enable regular monitoring in hydrocephalus patients.

In particular, non-invasive measurements in the external ear canal of middle-ear transmissions, including lowfrequency distortion-product otoacoustic emissions (DPOAEs) 
and reflectance, appear to depend on ICP (Voss et al., 2006). Postural changes in healthy humans cause systematic changes in ICP. The effects of postural changes, and presumably ICP changes, on DPOAE magnitude, DPOAE angle, and the reflectance measures of power reflectance and transmittance have been measured. In general, DPOAE magnitudes decrease with increased ICP at frequencies from 500 to $2000 \mathrm{~Hz}$, and the corresponding angle shows systematic changes with ICP in a given individual. Low-frequency power reflectance appears to increase systematically with increased ICP, while the corresponding transmittance decreases systematically.

Paulat et al. (1999) and Stettin et al. (2011) investigated whether ICP can be assessed by measuring infrasonic emissions from the TM. In a total of 83 measurements, the changes in ICP that were observed in response to different types of stimulation were detected in noninvasively and invasively acquired waveforms. Low ICP was associated with an initial high peak and later peaks were associated with smaller amplitudes. High ICP was associated with a marked decrease in the number of peaks and in the difference between the amplitudes of the initial and final peaks. However, the assessment of infrasonic emissions is not yet quantitative.

Zou et al. (2008) analyzed 11 experiments from 3 normal mongrel dogs with normal ICP. Intracranial pressures were manipulated by bolus injections, infusions, or the withdrawal of cerebrospinal fluid (CSF). The relationship between the ICP waveform and ABP can be quantitatively characterized using a newly developed technique in systems analysis, the time-varying transfer function. This technique utilizes the $\mathrm{ABP}$ as an input signal, composed of multiple frequencies, and translates it into an output ICP according to the transfer function imposed by the intracranial system on the input signal. The transfer function can change with time and in response to physiological manipulations. During periods of normal ICP, the gain of the transfer function has a deep notch centered at or near the cardiac frequency. In systems terms and under normal conditions, the intracranial compartment appears to act as a notch filter attenuating the cardiac frequency input relative to other frequencies. Periods of elevated ICP correspond to a suppression of the notch, and the notch is then restored when the ICP returns to normal.

In this study, a low-frequency microphone was embedded in an earplug that was placed into the external auditory meatus in an airtight manner. The microphone measured vital infrasound pressure in the occluded ear canal. In this manner, the relationship between TM umbo displacement and ECP was determined. The ECP waveform detected by the earplug yielded a pulsatile recording with a slow respiratory component superimposed on a biphasic recording that was synchronous with the cardiac cycle, and the ECP respiratory waveform was correlated with the instantaneous heart rate. The relationship between the infrasound pressure (ECP) from the TM and the CAP, which was detected using a stethoscope fitted with a low-frequency microphone, was quantitatively characterized using a newly developed systems analysis technique, the time-varying transfer function. The ECP and CAP waves were represented as a series of sinusoidal waves consisting of the fundamental cardiac frequency and its multiple harmonics (discrete multiples of the cardiac frequency). Last, various fatigue-related risk factors affected the mean magnitudes of the pressure readings and delayed transfer functions between CAP and ECP in the study population. These factors were systolic blood pressure, salivary amylase activity, age, sleep, postural changes, chronic fatigue, and pulse rate.

\section{RATIONALE}

We proposed to create a pressure chamber model in the occluded ear canal using a specialized earplug for electroacoustic transduction (Matsubara, 1999). In this model, the TM and a condenser microphone are coupled to the same cavity, and a known sinusoidal motion is imparted to the TM by means of the malleus. The pressure in the cavity can be calculated from the degree of umbo displacement. The expression for the pressure can be derived by considering the essentially adiabatic process. Differentiating the expression $P V^{\gamma}=$ a constant, yields for small displacements

$$
\delta P=-\gamma P_{0} S \delta L / V
$$

where $P=$ the sum of the sound pressure and atmospheric pressure $P_{0} ; V=$ cavity volume; $\gamma=$ the ratio of specific heats for the gas, which is equal to 1.403 for ambient air under normal conditions $\left(20^{\circ} \mathrm{C}, 760 \mathrm{mmHg}\right) ; \delta P=$ the sound pressure of the cavity; $S=$ the cross-sectional area of the $\mathrm{TM}$; and $\delta L=$ umbo displacement. A pistonphone model based on Eq. (1) is valid under the conditions that (i) the walls of the cavity are perfectly rigid, (ii) the cooling effect of the walls is negligible, and (iii) standing wave patterns do not exist in the gas (Koidan, 1954).

Stinson and Lawton (1989) studied the geometry of the ear canals of 15 volunteers. The average cross-sectional area was $S=41.8 \mathrm{~mm}^{2}$, the average length was $l_{\mathrm{all}}=30.8 \mathrm{~mm}$, and the average canal volume was $V_{\text {all }}=1014 \mathrm{~mm}^{3}$. Therefore, the average cavity volume in the occluded ear canal with an earplug inserted into the ear canal (length $l=14 \mathrm{~mm}$ ) was $V=702 \mathrm{~mm}^{3}$. Figure 1 shows the sound pressure level (SPL) of the occluded ear canal cavity as a function of applied umbo displacement for the pistonphone model (dashed line), based on Eq. (1). The SPL response of the

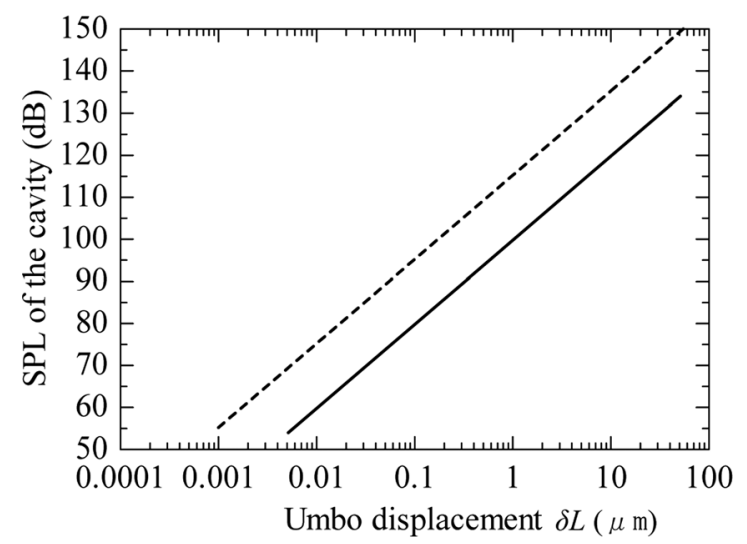

FIG. 1. The relationship between the SPL (dB) of the cavity and umbo displacement $\delta L(\mu \mathrm{m})$. Dashed line: the pistonphone model based on Eq. (1). Continuous line: the prediction model based on the compliance of 0.0215 $\mathrm{mm}^{3} / \mathrm{Pa}$ found by von Békésy (1936). 
cavity to the applied umbo displacement, according to Eq. (1), was approximately a linear function from $0.001 \mu \mathrm{m}$ to $100 \mu \mathrm{m}$. The compliance is defined as the volume displacement per unit pressure load. A compliance of $0.0047 \mathrm{~mm}^{3} /$ $\mathrm{Pa}$ is obtained using the pistonphone model. von Békésy (1936, 1941) has performed acoustic compliance measurements at the $0.1 \mathrm{kPa}$ root mean square SPL in the frequency range between $5 \mathrm{~Hz}$ and $50 \mathrm{~Hz}$. At $5 \mathrm{~Hz}$, von Békésy observed a compliance of $0.0215 \mathrm{~mm}^{3} / \mathrm{Pa}$ (continuous line: see Fig. 1). The assumptions (a), (b), and (c) of the pistonphone do not apply to the external auditory canal.

Thalen et al. (2002) measured the inner ear pressure in the scala tympani with a micropipette during square wave pressure manipulation of the intracranial compartment of a guinea pig, and subsequently, of the external ear canal in the same animal. As expected, the combination of the cochlear aqueduct and the inner ear behaves as a low-pass filtering system for intracranial pressure manipulation and as a complementary high-pass system for ear canal pressure manipulation. Time constants for pressure equalization were on the order of seconds and depended on the direction of flow through the cochlear aqueduct. Pressure equalization curves could not be fitted to a single exponential function; more complicated functions were needed to obtain good fits, demonstrating that the pressure equalization process is nonlinear. This means that the flow resistance of the cochlear aqueduct is not a constant value (Thalen et al., 2004). The cochlear aqueduct is a canal with dynamic properties and may play a role in the complicated process of inner ear pressure regulation. The cochlear aqueduct connects the scala tympani to the subarachnoid space and is the main pressure equalization canal for the inner ear. Increases in inner ear volume and pressure are thought to cause clinical symptoms such as vertigo, tinnitus, and fluctuating hearing loss.

\section{METHODS}

\section{A. Subjects}

A total of 40 subjects, ranging in age from 19 to 57 years participated in this study. Table I reports the subjects' ages and genders. All subjects were recruited from a database of potential research subjects maintained at Gunma Factory of Ichikawa Co. Ltd. Subjects were paid for their participation. After obtaining informed consent and administering a questionnaire consisting of 13 items that elicited psychosomatic symptoms (Japan Industrial Safety and Health Association, 2002), we questioned the subjects about their sleeping schedules, performed sphygmomanometry (EW243, Panasonic, Kadomashi, Osaka, Japan; systolic blood pressure, diastolic blood pressure, and beats per minute) and measured salivary amylase activity (Salivary Amylase Monitor, CM-21, Nipro Co. Japan; Yamaguchi et al.,

TABLE I. Number of subjects classified by age and gender.

\begin{tabular}{lcccccc}
\hline \hline Age & 19 & $20-29$ & $30-39$ & $40-49$ & $50-57$ & Total \\
\hline Male & 1 & 8 & 3 & 7 & 3 & 22 \\
Female & 0 & 1 & 6 & 8 & 3 & 18 \\
& 1 & 9 & 9 & 15 & 6 & 40 \\
\hline \hline
\end{tabular}

2006). We used a scaled survey instrument to assess the workers for their general level of fatigue and the presence of any psychosomatic symptoms. The survey consisted of 13 items covering a wide range of symptoms including irritability, anxiety, loss of equanimity, depression, insomnia, nausea, loss of concentration, carelessness, sleepiness at work, and loss of energy. The response options on the survey of psychosomatic symptoms were based on frequency and were scored from "almost never" $=0$ to "very often" $=3$. Total scores ranged from 0 to 39 , with a higher score indicating a greater burden of psychosomatic symptoms. The survey showed acceptable levels of internal consistency and reliability (Cronbach's alpha $=0.90)$ in this study sample.

Measuring the effect of postural changes on cerebral hemodynamics required 3 min per subject: the subject sat on a bed for $1 \mathrm{~min}$, lay in a supine position for $1 \mathrm{~min}$, and then sat again for $1 \mathrm{~min}$. Each subject was assessed in this manner three times each day in the morning, afternoon and evening, five days per week. The data were measured from Nov. 7 , 2011 to Dec. 9, 2011.

\section{B. Measurement equipment}

A continuous, non-invasive method and apparatus were developed to measure the transfer function between ECP (infrasonic emissions from the TM) and CAP (a rushing sound that can be auscultated by placing a stethoscope over the carotid artery), which is determined by various hemodynamic parameters [see Fig. 2(a)]. A microphone was embedded in an earplug that is capable of sealing the outer entrance to an individual's ear canal such that arterial blood pressure changes adjacent to the sealed ear canal produce changes in the air pressure in the cavity. The other apparatus is a stethoscope that is fitted with a low frequency microphone to detect carotid artery pressures. Different responses to the ECP were obtained from the earplug microphone and an earlobe sensor (Model PL-6000, Cateye, Higashisumiyoshiku, Osaka, Japan), which produces an ear pulse oximeter wave (EPOW), and the responses from these two devices were compared.

A condenser microphone was attached to an earplug with an air chamber to measure the sound wave from the ear canal caused by pulse beats, breathing, and intracranial pressure.

A stethoscope helps to transmit the sound to the surface of the skin where images of the carotid arteries and pulse waveforms can be obtained.

Primo markets an electret condenser microphone with a low-frequency response as low as $0.5 \mathrm{~Hz}$ (Model EM162S5A, Primo, Nishitamagun Mizuhomachi, Tokyo). Figure 2(b) shows the circuit diagram without a coupling condenser used to obtain the measurement, and Fig. 2(c) shows the frequency response. The electret condenser microphone has a diameter of $10.0 \mathrm{~mm}$ and a height of $5.4 \mathrm{~mm}$. The frequency response remains flat from $0.5 \mathrm{~Hz}$ to $100 \mathrm{~Hz}$ within $2.0 \mathrm{~dB}$. Measurements using the condenser were carried out according to the pressure calibration method in a closed loudspeaker cabinet at a constant SPL of $94 \mathrm{~dB}$. The microphone's level of sensitivity was $-41.0 \mathrm{~dB}$ re $1 \mathrm{~V} / \mathrm{Pa}$ at $100 \mathrm{~Hz}$.

The preamplifier is intended for use with capacitive sensors, including the capacitive electret film type sensor, one of 
(a)

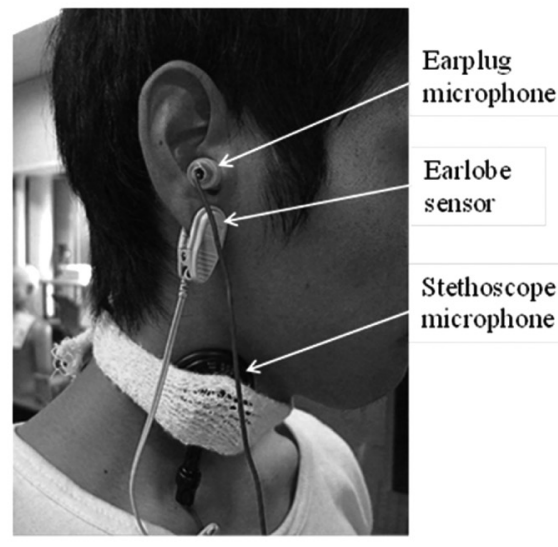

(b)

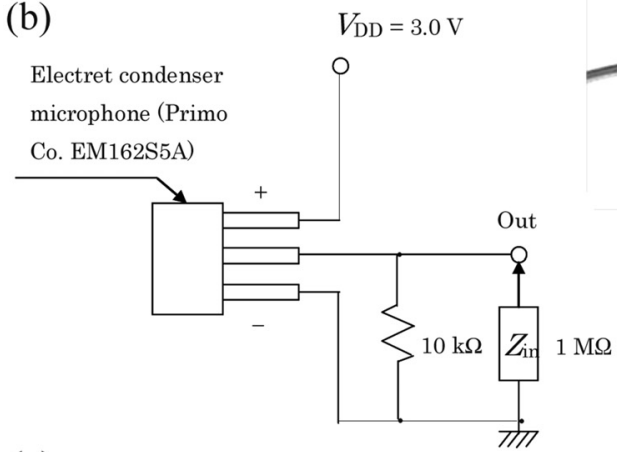

(c)

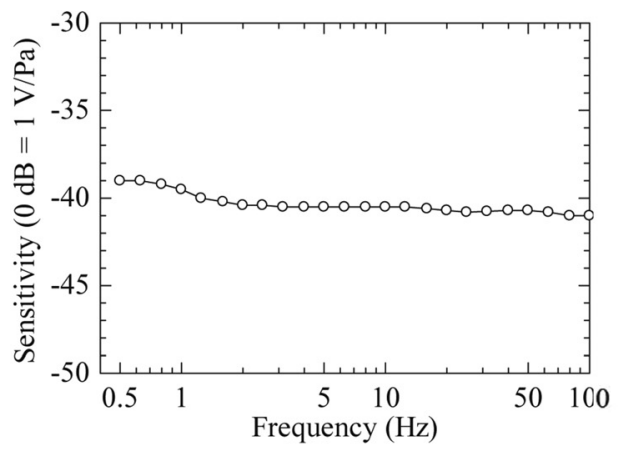

(d)

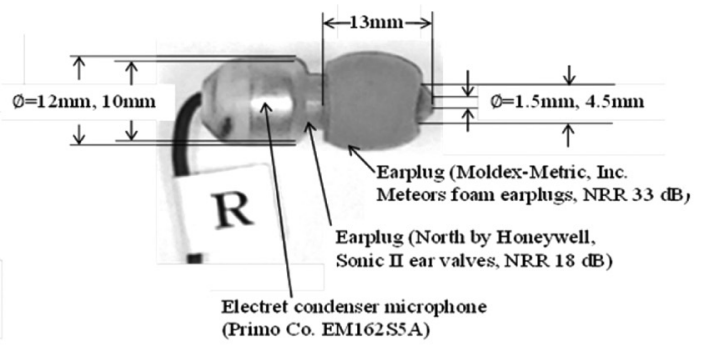

(e)

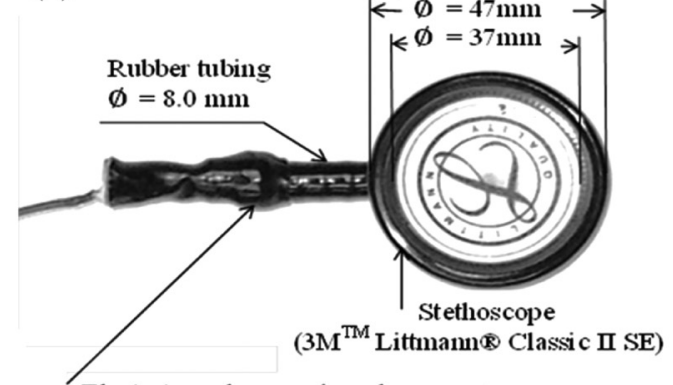

Electret condenser microphone (Primo Co. EM162S5A)

(f)

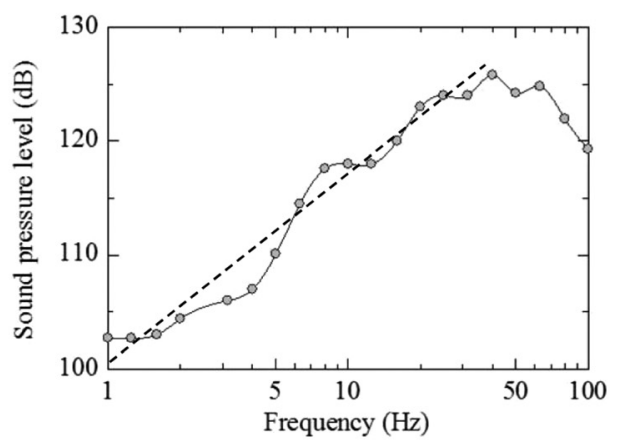

FIG. 2. Continuous, non-invasive method and apparatus (newly developed microphone systems for detecting vital infrasonic emissions) used to measure the transfer function between the CAP and ECP signals as a function of various hemodynamic parameters. (a) Measurement equipment, (b) the circuit diagram of a microphone with a low-frequency response, (c) the frequency response of the microphone, (d) an earplug microphone, (e) a stethoscope microphone, and (f) the frequency response of the stethoscope (constant displacement; $\delta L=10.0 \mu \mathrm{m})$. the most sensitive and accurate sensors available. Both direct current (DC)-coupled and alternating current (AC)-coupled conventional preamplifiers are available. However, DCcoupled preamplifiers do not always suppress drift noise and vibration, and AC-coupled preamplifiers require a large capacitor to expand the frequency range. Therefore, a 12-bit, $100 \mathrm{kHz}$ analog-to-digital (A/D) converter was selected. This A/D converter (TUSB 0412ADSM, Turtle Industry Co., Tsuchiurashi, Ibaraki, Japan) has a DC-coupled preamplifier with an upper range of $500 \mathrm{kHz}$ (the unipolar single-ended input range).

Figures 2(d) and 2(e) show the microphone system that was developed to detect vital infrasonic emissions: Fig. 2(d) shows an earplug microphone, a microphone integrated into an earplug; Fig. 2(e) shows a stethoscope microphone, a microphone fitted to the flexible rubber tube connected with the bell/diaphragm of the stethoscope (Classic II SE, $3 \mathrm{M}^{\mathrm{TM}}$ Littmann $^{\circledR}$, St. Paul, MN). The earplug microphone was made up of three parts: the electret condenser microphone with a shielding wire $(\phi=1.6 \mathrm{~mm}$, length $=3.5 \mathrm{~m})$ and components from two different models of earplugs (Sonic II ear valves,
North by Honeywell, Morris Township, NJ, and Meteor foam earplugs, Moldex-Metric, Inc., Culver City, CA). The latter earplug is made of soft, slow-recovery foam and its contoured shape molds easily to the ear canal. The earplug microphone was inserted by rolling the foam earplug component into its smallest diameter, placing the tapered end quickly into the ear canal and holding it in place until it fully expanded.

Abella et al. (1992) evaluated stethoscope acoustics using a sound frequency generator and an active artificial ear. Their results showed that the bell and diaphragm of a given stethoscope usually have different transmission characteristics, particularly at low frequencies $(37.5-112.5 \mathrm{~Hz})$. The Littmann Classic II is an exception, but the sound transmission characteristics in the infrasonic frequency range (1$20 \mathrm{~Hz}$ ) have not been reported. Therefore, the model's response to a range of frequencies $(1-100 \mathrm{~Hz})$ was evaluated. Measurements were taken to ensure that displacement (amplitude $\delta L=10 \mu \mathrm{m}$ ) took place at the center of the stethoscope diaphragm, which was excited using a vibration sensor (PV-09 A, RION, Kokubunjishi, Tokyo: sensitivity: 
$68.09 \mathrm{pC} / \mathrm{G}$; driving area: $303.5 \mathrm{~mm}^{2}$; diameter: $13.9 \mathrm{~mm} \phi$; length: $30.0 \mathrm{~mm}$ ) screw-clamped to a vibrator ( $\mathrm{Vt7}$, Acouve Laboratory, Chiyodaku, Tokyo: body diameter: $80 \mathrm{~mm} \phi$ ). A sine wave function generator (FG-143, NF circuit design, Chuoku, Tokyo) and a power amplifier (CA-S10, Flying, Hamamatsu Nishiku, Shizuoka, Japan) were used to produce a sine wave vibration. The acceleration level was measured using a charge amplifier (Model 4006, Showa Sokki, Chiyodaku, Tokyo). A rubber coupler placed between the chest piece of the stethoscope and the vibrator was used as the supported edge, and a $1 \mathrm{~mm}$ thick piece of silicon rubber was placed between the stethoscope's diaphragm and the vibration sensor as a cushioning material. Figure 2(f) shows the frequency response of the stethoscope when the center of the diaphragm was vibrated with constant displacement $(\delta L=10 \mu \mathrm{m})$. The resonant frequency was approximately 40 $\mathrm{Hz}$, and the SPL changed from $102.7 \mathrm{~dB}$ to $123.0 \mathrm{~dB}$ (slope upward at approximately $20 \mathrm{~dB} /$ decade; dashed line) from $1 \mathrm{~Hz}$ to $20 \mathrm{~Hz}$.

Responses from the ECP were obtained from the earplug microphone and an earlobe sensor (Cateye Model PL-6000), and the responses from these two devices were compared. The instantaneous heart rate signal was obtained from the EPOW reading using the peak detection algorithm.

These signals were simultaneously recorded and digitized at a sampling rate of $1000 \mathrm{~Hz}$ using a data acquisition system. The system consisted of the 4-channel A/D converter and a notebook computer (Panasonic CF-F10AWHDS). The A/D universal serial bus (USB) interface converter is easily connected to the computer's USB port.

\section{Transfer function between CAP and ECP}

We investigated whether transfer functions, which were calculated based on the fast Fourier transform (FFT) of input (CAP) and output (ECP) signals from normal subjects, can provide more useful information than absolute ICP values.

The application of system identification methods to ICP analysis dates back to 1980, when Chopp and Portnoy (1980) described the cerebrovascular system as a black-box model. In their model, ABP was defined as the chief input to the system, and ICP was considered to be the output response to that stimulus. This type of investigation is based on the belief that intracranial compliance can demonstrate frequency dependence. This implies that there may be a particular response mechanism within the cerebrovascular system, i.e., filtering or convolution, that handles different frequencies differently (Zou et al., 2008). In the conventional, nonparametric modeling of the transfer function between ABP and ICP, which is an FFT-based method, both ABP and ICP are transferred to the frequency domain. ICP and $\mathrm{ABP}$ are expressed here as a series of sinusoidal waves, including the fundamental cardiac component and its multiple harmonics. The transfer function can then be calculated as the ratio of the cross-spectrum of the input and output signals $\left[P_{x y}(\omega)\right]$ to the auto-spectrum of the input $\left[P_{x x}(\omega)\right]$ in stochastic cases, or as the ratio between the Fourier transforms of the output and input signals $[Y(\omega)$ and $X(\omega)]$ in deterministic cases. An estimate of the transfer function can be obtained from

$$
H(\omega)=\frac{P_{x y}(\omega)}{P_{x x}(\omega)}=\frac{X^{*}(\omega) Y(\omega)}{X^{*}(\omega) X(\omega)}=\frac{Y(\omega)}{X(\omega)},
$$

where $X^{*}(\omega)$ is the complex conjugate of $X(\omega)$. The amplitude of the transfer function determines how much pressure is transmitted through the cerebrovascular bed at a given frequency, while its phase indicates the phase shift for that specific frequency component (Piper et al., 1990; Zou et al., 2008).

Portnoy and Chopp (1981) conducted experiments on cats with elevated ICP. They reported an increase in the ICP waveform pulse and a simultaneous increase in the amplitude of the transfer function at the fundamental cardiac frequency during periods of arterial hypercapnia and hypoxia. Further investigation by Portnoy et al. (1982) showed that in cases of low ICP (ICP $<7 \mathrm{mmHg}$ ) in dogs, an attenuation of amplitude occurs in the transfer function at the fundamental frequency, which appears to normalize during hypercapnic episodes or intraventricular infusion. They attributed this attenuation to the functional autoregulatory tone of the cerebral precapillary resistance vessels and interpreted it as a characteristic of cerebral autoregulation.

Employing similar methods, Takizawa et al. (1987) demonstrated a positive correlation between elevated ICP and the amplitude of the first four cardiac harmonics in both the ICP waveform and the transfer function. Their study also showed that the ICP becomes more like a single sine wave when ICP levels approach $50 \mathrm{mmHg}$.

Piper et al. (1990) adopted the system analysis method of Portnoy and Chopp (1981) and applied it to a clinical study of cerebrovascular pressure transmission. Analyzing 1500 ICP recordings collected from 30 patients with traumatic brain injury, he identified four different patterns in the transfer function between ABP and ICP: low amplitude at both low and high frequencies, low amplitude at low frequencies and high amplitude at high frequencies, high amplitude at low frequencies and low amplitude at high frequencies, and high amplitude at both low and high frequencies.

According to his observations, both groups with elevated amplitudes at the fundamental cardiac frequency were associated with elevated ICPs, while the two remaining groups were associated with ICPs of less than $15 \mathrm{mmHg}$. His further investigations in cats demonstrated that the three different mechanisms that mediate active arteriolar vasodilation, the loss of autoregulatory vascular tone, and the reduction of cerebrovascular transmural pressure (Piper et al., 1993) are involved in increasing the amplitude of the transfer function at the fundamental cardiac frequency. These mechanisms were distinguished from one another by taking into account the phase shifts between the fundamental cardiac frequency components in ABP and ICP. In this experimental model, active vasodilation was associated with a negative phase shift, while a decreased transmural pressure was not associated with a phase shift.

Although various groups have studied cerebrovascular pulse transmission, the appropriateness of these techniques has been widely debated. The use of a transfer function assumes that a linear transmission occurs from the input to the output signals. This assumption may not be valid for 
biological signaling, particularly in various disease states (Czosnyka et al., 2007). Another drawback of using these conventional methods is that they are time-invariant and are, therefore, not capable of tracking signal evolution over time. This disadvantage may be overcome with the use of timevarying transfer function models that utilize short segments of time series data during which signals can be assumed to be stationary. Time-varying transfer function models are purported to be capable of identifying inherent, nonstationary dynamics in biomedical systems (Zou et al., 2008).

Recently, a time-varying transfer function utilizing autoregressive-moving-average modeling was applied by Zou and colleagues (2008) to ICP and ABP signals collected in dogs. Their investigations revealed the existence of a deep notch in the amplitude of the transfer function, centered at or near the cardiac frequency for normal ICP. This notch was suppressed when ICP was increased with a bolus injection. These results are similar to those reported by Portnoy et al. (1982), who proposed that a pulsation absorber may exist in the intracranial systems of animals for which the target frequency appears to approximate the cardiac frequency. The free movement of cerebrospinal fluid was proffered as one possible absorbing mechanism.

The ICP signal includes a mean pressure component and pressure pulsations associated with the cardiac and respiratory cycles. The ICP signal can be regarded as a composite wave that contains the following components:

(i) Pulse waveform: The pulse waveform includes several harmonics with a fundamental frequency that is equal to the heart rate. It has been argued that the amplitude of the fundamental component is useful in evaluating certain indices of cerebrospinal dynamics (Czosnyka et al., 2007).

(ii) Respiratory waveform: The respiratory waveform is related to the frequency of the respiratory cycle (12 to 30 cycles per minute).

(iii) Interaction among cardiac and respiratory components: The existence of a cardiorespiratory effect on blood pressure has been known for a long time, and the effects of respiration on heart rate have been studied by a number of groups (Smelyanskiy et al., 2005; Zwiener et al., 1995), but little attention has been given to the effects of respiration on intracranial pressure.

(iv) Slow waves: Slow waves are not precisely defined. All components that have a spectral representation within the frequency limits of 0.05 to $0.0055 \mathrm{~Hz}$ (lasting from $20 \mathrm{~s}$ to $3 \mathrm{~min}$ ) can be classified as slow waves (Czosnyka et al., 2007).

Figures 3(a) and 3(b) depict the short-time Fourier transform from $10 \mathrm{~min}$ of recorded CAP and right ECP signals that were collected from a healthy subject. Note that the CAP and ECP spectra contain slow waves with frequencies less than $0.1 \mathrm{~Hz}$, a respiratory component $\left(f_{R}\right)$ from $0.2 \mathrm{~Hz}$ to $0.5 \mathrm{~Hz}$ and a cardiac component $\left(f_{C}\right)$ of approximately $1 \mathrm{~Hz}$, along with their higher harmonics. The CAP and ECP spectra also include the interactions between the two latter components, which are located at $f_{C}-f_{R}, f_{C}-2 f_{R}, f_{C}+f_{R}$, and so on. Figures 3(a) and 3(b) suggest that the assessment of infrasonic emissions (ECP) will someday be suitable both as a screening tool and for the continuous monitoring of ICP in an intensive care environment (Stettin et al., 2011).

For ECP signal analysis, this study employed a moving average filter system to separate two bio-impedance signal components: the cardiac and respiratory signals. The moving average filter operates by averaging a number of points from the input signal to produce each point in the output signal. In equation form

$$
y(i)=\frac{1}{M} \sum_{j=-(M-1) / 2}^{(M-1) / 2} x(i+j),
$$

where $x()$ is the input signal, $y()$ is the output signal, and $M$ is the number of points used to calculate the average.

The frequency response of the moving filter is mathematically described by the Fourier transform of the rectangular pulse

$$
H(f)=\frac{\sin (\pi f M)}{M \sin (\pi f)} .
$$

The moving average is a very poor low-pass filter due to its slow roll-off and poor stopband attenuation. However, the moving average filter has notch characteristics. The notch in the frequency response of the filter can be made to automatically track the drift of a cardiac signal frequency. Therefore, the heart rate (the number of heartbeats per unit of time) was used to determine the $M$ value and was measured from the pulse oximeter and the peak detection algorithm.

Additionally, spectral analysis (sampling frequency $1000 \mathrm{~Hz}$, Hanning window, FFT points; 4096, 50\% overlap) detected one fundamental frequency and multiple harmonic components, and transfer functions were evaluated at those discrete frequencies only. For example, the magnitude of the function at the fundamental frequency is calculated as the ratio of the amplitude of the fundamental spectral frequency $\left(A_{\text {out }} / A_{\text {in }}\right)$. The magnitudes at each of the harmonic components were estimated in the same way.

\section{Statistics}

First, a multiple regression analysis (MRA) (Field, 2010) was performed to explore the relationships between the measured transfer function and the various parameters: age, gender, systolic blood pressure (SBP), diastolic blood pressure, pulse rate (beats per minute), sleeping time, salivary amylase activity (SAA), and accumulated fatigue (general fatigue and the presence of any psychosomatic symptoms). PASW version 18.0 for Windows (IBM SPSS Statistics, New York) was used for the statistical analysis of the results. To reduce the number of parameters, we performed a principal component analysis (PCA) prior to the MRA. The PCA clusters the parameters into factors. We used a varimax rotation to maximize the dispersion of loadings within factors (Field, 2010). This approach aims to load a smaller number of variables onto each factor, resulting in easier to interpret clusters of factors.

Second, a discriminant function analysis was used to classify cases according to the values of the transfer function. 

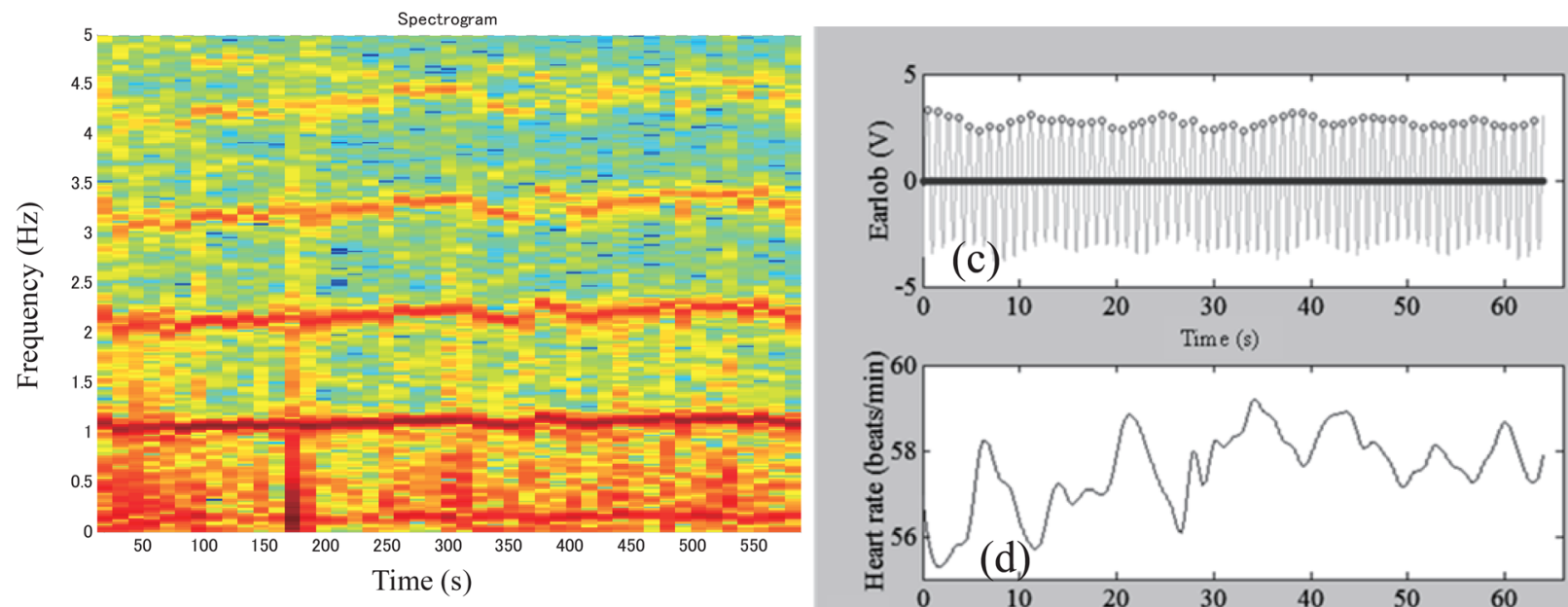

(a)
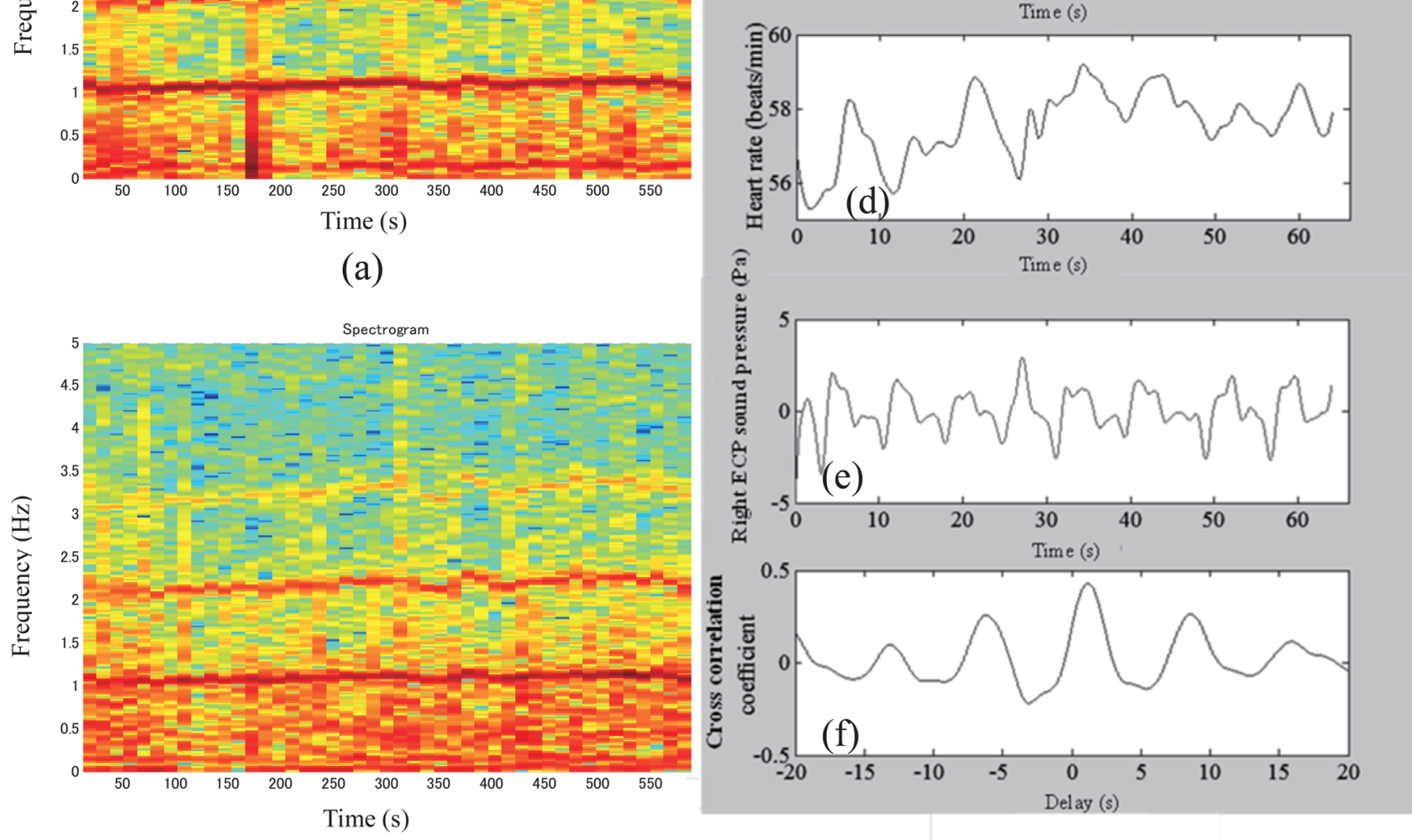

(b)

FIG. 3. (Color online) Short-time Fourier transform (Spectrogram) over ten min for (a) CAP and (b) right ECP signals collected from a normal subject: (a) the spectrogram of the CAP signal, (b) the spectrogram of the right ECP signal. The CAP and ECP spectra contain slow waves with frequencies lower than $0.1 \mathrm{~Hz}$, a respiratory component $\left(f_{R}\right)$ from $0.2 \mathrm{~Hz}$ to $0.5 \mathrm{~Hz}$, a cardiac component $\left(f_{C}\right)$ of approximately $1 \mathrm{~Hz}$, and their higher harmonics. The relationship between the heart rate (beats/min) and the respiratory signal of the right ECP for a healthy subject: (c) the pulse oximeter wave (EPOW) measured from the earlobe and the peak detection algorithm, (d) the instantaneous heart rate signal, (e) the respiratory signal of the right ECP, and (f) the cross-correlation coefficient between the instantaneous heart rate signal and the respiratory signal of the right ECP.

\section{RESULTS}

\section{A. SPLs of CAP and ECP measured in the sitting and supine positions in forty healthy subjects}

SPLs $(\mathrm{dB})$ associated with CAP and ECP signals were measured for forty healthy subjects in the sitting and supine positions, and weekly average SPLs were calculated for each subject (6 to 15 measurements per week). The mean measured SPLs with standard deviations (averaged over the entire study population) are shown in Table II and are separated into the circulatory and respiratory components of CAP and ECP. For the circulatory component: (i) the mean SPL of CAP was $113.7 \mathrm{~dB}$ in the sitting position and $119.0 \mathrm{~dB}$ in the supine position, meaning that the postural change led to

TABLE II. The mean and standard deviation (SD) values of the CAP and ECP signals for the circulatory and respiratory systems in response to postural changes.

\begin{tabular}{|c|c|c|c|c|c|c|c|c|c|c|c|c|}
\hline \multirow[b]{3}{*}{ Position } & \multicolumn{6}{|c|}{ Circulatory system } & \multicolumn{6}{|c|}{ Respiratory system } \\
\hline & \multicolumn{2}{|c|}{$\begin{array}{l}\text { SPL }(d B) \\
\text { of CAP }\end{array}$} & \multicolumn{2}{|c|}{$\begin{array}{l}\text { SPL }(d B) \text { of the } \\
\text { right ECP }\end{array}$} & \multicolumn{2}{|c|}{$\begin{array}{l}\text { SPL }(d B) \text { of the } \\
\text { left ECP }\end{array}$} & \multicolumn{2}{|c|}{$\begin{array}{l}\text { SPL }(d B) \\
\text { of CAP }\end{array}$} & \multicolumn{2}{|c|}{$\begin{array}{l}\mathrm{SPL}(\mathrm{dB}) \text { of the } \\
\text { right } \mathrm{ECP}\end{array}$} & \multicolumn{2}{|c|}{$\begin{array}{l}\text { SPL }(d B) \text { of the } \\
\text { left ECP }\end{array}$} \\
\hline & Mean & SD & Mean & SD & Mean & SD & Mean & SD & Mean & SD & Mean & SD \\
\hline First sitting & 113.7 & 3.0 & 98.4 & 5.1 & 96.8 & 5.5 & 101.4 & 3.3 & 83.5 & 5.4 & 81.7 & 4.7 \\
\hline Supine & 119.0 & 4.2 & 97.1 & 4.2 & 95.1 & 5.0 & 102.7 & 3.6 & 80.8 & 4.9 & 78.0 & 4.5 \\
\hline Second sitting & 113.7 & 3.2 & 97.7 & 6.0 & 96.2 & 5.7 & 101.7 & 3.5 & 82.1 & 5.5 & 81.2 & 5.3 \\
\hline
\end{tabular}


TABLE III. The mean and SD values of the cross-correlation function between heart rate variability and the respiratory component of each ECP signal during postural changes.

\begin{tabular}{|c|c|c|c|c|c|c|c|c|}
\hline \multirow[b]{3}{*}{ Position } & \multicolumn{4}{|c|}{$\begin{array}{l}\text { Cross-correlation function between heart rate variability } \\
\text { and respiratory component of the right ECP }\end{array}$} & \multicolumn{4}{|c|}{$\begin{array}{l}\text { Cross-correlation function between heart rate variability } \\
\text { and respiratory component of the left ECP }\end{array}$} \\
\hline & \multicolumn{2}{|c|}{ Coefficient } & \multicolumn{2}{|c|}{ Delay (sec) } & \multicolumn{2}{|c|}{ Coefficient } & \multicolumn{2}{|c|}{ Delay (sec) } \\
\hline & Mean & $\mathrm{SD}$ & Mean & $\mathrm{SD}$ & Mean & SD & Mean & $\mathrm{SD}$ \\
\hline First sitting & 0.341 & 0.077 & 1.568 & 4.346 & 0.279 & 0.046 & -0.812 & 5.103 \\
\hline Supine & 0.397 & 0.077 & 0.665 & 2.400 & 0.366 & 0.082 & 0.329 & 2.276 \\
\hline Second sitting & 0.378 & 0.076 & -0.101 & 2.261 & 0.356 & 0.082 & 0.234 & 1.965 \\
\hline
\end{tabular}

$\mathrm{a}+5.3 \mathrm{~dB}$ difference; (ii) the mean SPL of the right ECP was approximately $98.0 \mathrm{~dB}$ in the sitting position and $97.1 \mathrm{~dB}$ in the supine position for a $-0.9 \mathrm{~dB}$ difference; (iii) and the difference between the mean right- and left-sided ECPs was approximately $1.7 \mathrm{~dB}$. For the respiratory component: (i) the mean SPL of CAP was $101.4 \mathrm{~dB}$ in the sitting position and $102.7 \mathrm{~dB}$ in the supine position, representing $\mathrm{a}+1.3 \mathrm{~dB}$ difference resulting from the postural change; (ii) the mean SPL of the right ECP was approximately $83.0 \mathrm{~dB}$ in the sitting position and $80.8 \mathrm{~dB}$ in the supine position for a difference of $-2.2 \mathrm{~dB}$; (iii) and the difference between the mean right- and left-sided ECPs was approximately $1.8 \mathrm{~dB}$.

\section{B. The relationship between the heart rate variability and the respiratory signal of the ECP}

Heart rate variability (HRV) indices provide a noninvasive assessment of cardiovascular control mechanisms (Tripathi, 2004). Significant variations in heart rate occur during normal day-to-day activities. The heart rate slows during sleep and increases upon standing up from a seated position, as well as during stressful situations and exercise. Even during quiet rest, significant fluctuations in heart rate occur. There has been a great deal of recent interest in quantifying HRV because of the growing body of evidence that HRV is reduced in certain disease states (e.g., congestive heart failure, myocardial infarction, diabetes). This reduction most likely results from a failure in one or more of the control systems regulating the heart rate. Figures 3(c)-3(f) show the relationship between HRV and the respiratory component of ECP for a healthy subject. Figure 3(c) shows the EPOW and the peak detection algorithm, Fig. 3(d) shows the instantaneous heart rate signal, Fig. 3(e) shows the respiratory signal of the right ECP [see Fig. 3(b)], and Fig. 3(f) shows the cross-correlation coefficient between the instantaneous heart rate signal and the respiratory signal of the right ECP. A comparison between Figs. 3(d) and 3(e) indicates that HRV correlates with the respiratory signal, once the latter is extracted from the rest of the right ECP wave. As depicted in Fig. 3(f), the cross-correlation coefficient between HRV and the respiratory signal is 0.428 , and the delay time is $1.175 \mathrm{~s}$. Table III shows the mean and standard deviation (SD) of the cross-correlation function (the maximum coefficient and the delay time) between heart rate variability and the respiratory signal of each ECP measured in the sitting and supine positions in forty health subjects.

\section{Waveforms of ECP and CAP classified by systolic blood pressure}

Figure 4 shows the relationship between the average root mean squared sound pressure $(\mathrm{Pa})$ of the right ECP and the average SBP (mmHg) for each subject from the first and second seated readings. The correlation coefficient is 0.581 . The coefficient is significant at the 0.01 level.

Table IV shows the characteristics of three subjects selected for their SBP. Subject A had the lowest SBP of the 40 subjects, $B$ was a normal subject, and subject $C$ had the highest SBP. Figures 5(a)-5(c) show the waveforms of the circulatory system (continuous line) and the respiratory system (dashed line) for the right ECP readings for each subject, taken in the sitting position: Fig. 5(a) subject A, Fig. 5(b) subject B, Fig. 5(c) subject C. A comparison of the subjects revealed the following:

(i) The respiratory waveforms (dashed line) in Figs. $5(\mathrm{a})-5$ (c) range from $-0.18 \mathrm{~Pa}$ to $0.25 \mathrm{~Pa},-0.56 \mathrm{~Pa}$ to $0.39 \mathrm{~Pa}$, and $-3.66 \mathrm{~Pa}$ to $5.62 \mathrm{~Pa}$, respectively.

(ii) The circulatory waveforms (continuous line) in Figs. 5(a)-5(c) range from $-3.07 \mathrm{~Pa}$ to $2.06 \mathrm{~Pa},-8.95 \mathrm{~Pa}$ to $4.42 \mathrm{~Pa}$, and $-28.93 \mathrm{~Pa}$ to $19.97 \mathrm{~Pa}$, respectively.

(iii) The circulatory pulse waveforms mainly consist of three components: $P_{1}, P_{2}$, and $P_{3}$ as shown in Figs. $5(\mathrm{a})-5(\mathrm{c})$.

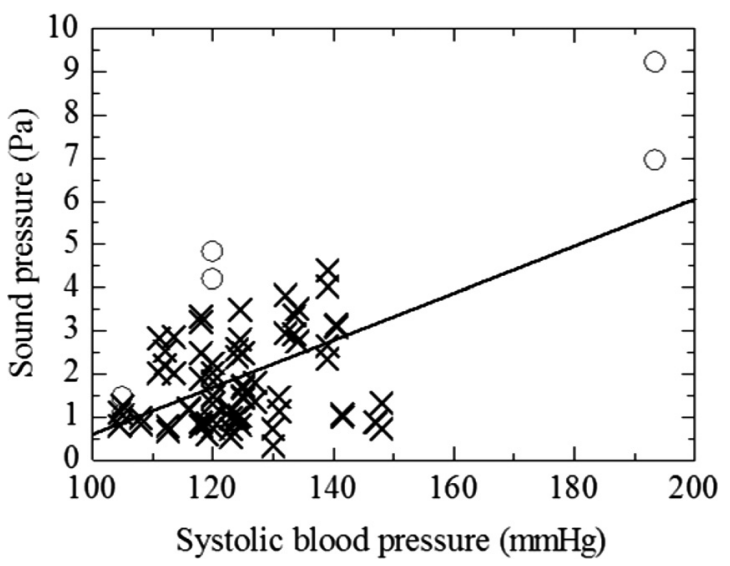

FIG. 4. The relationship between the root mean square sound pressure $(\mathrm{Pa})$ of the right ECP and the mean systolic blood pressure $(\mathrm{mmHg})$ for each subject for the first and second seated intervals. 
TABLE IV. Blood pressure characteristics of three selected subjects.

\begin{tabular}{lcccccc}
\hline \hline Subject & Gender & Age & Heart rate (beats/min) & Systolic blood pressure (mmHg) & Diastolic blood pressure (mmHg) & Salivary amylase activity (kIU/L) \\
\hline A & Female & 45 & 66 & 96 & 65 & 31 \\
B & Male & 25 & 72 & 111 & 75 & 120 \\
C & Male & 43 & 100 & 189 & 109 \\
\hline \hline
\end{tabular}

(iv) Under normal conditions as in Figs. 5(a) and 5(b), $P_{1}$ is the highest of the three waveform components.

(v) For Fig. 5(c) with the highest SBP, $P_{2}$ is higher than $P_{1}$.

Lundberg (1960) first introduced the ICP monitoring system and began investigating the ICP pulse waveform. ICP pulse waveforms are generated from a pressure wave that is transmitted through the cardiovascular system into the tissues within the intracranial cavity (Kirkness et al., 2000), including the choroid plexus where CSF is produced. The pulse waveform consists of three components: $P_{1}, P_{2}$, and $P_{3}$, as shown in Figs. 5(a)-5(c), respectively. The percussion wave $P_{1}$ reflects the pulsations of the choroid plexus, as transmitted from the cardiovascular system during systole. Under normal conditions, $P_{1}$ is the highest of the three waveform components. The tidal wave $P_{2}$ has a more variable shape and is indicative of the relative brain volume. The $P_{2}$ component is often elevated in response to a rapidly expanding mass lesion, such as a hematoma or an intracranial tumor (Kirkness et al., 2000; March, 2000). The dicrotic wave $P_{3}$
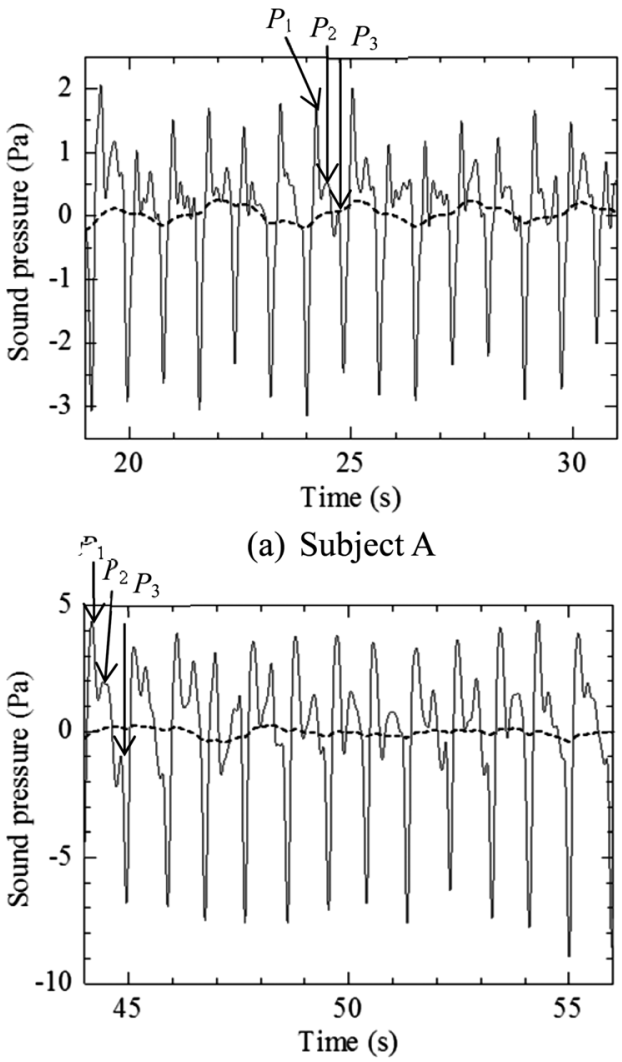

(b) Subject $\mathrm{B}$

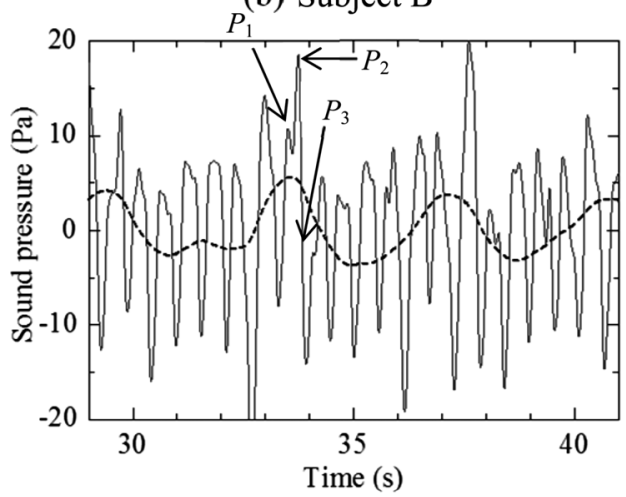

(c) Subject C

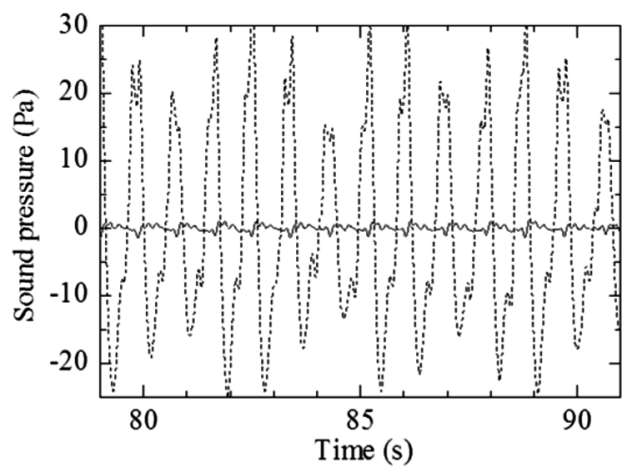

(d) Subject A

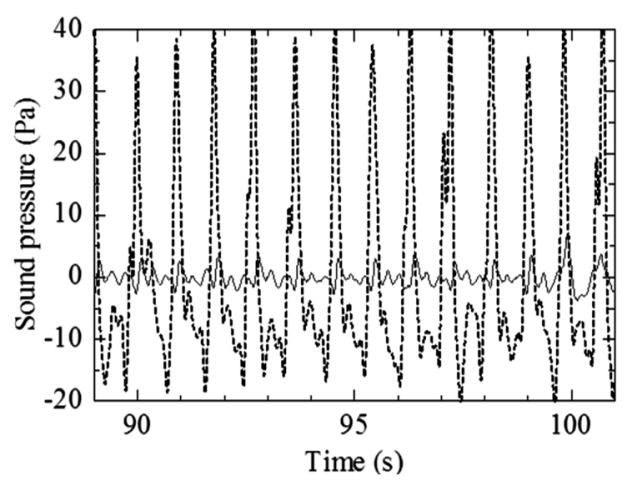

(e) Subject B

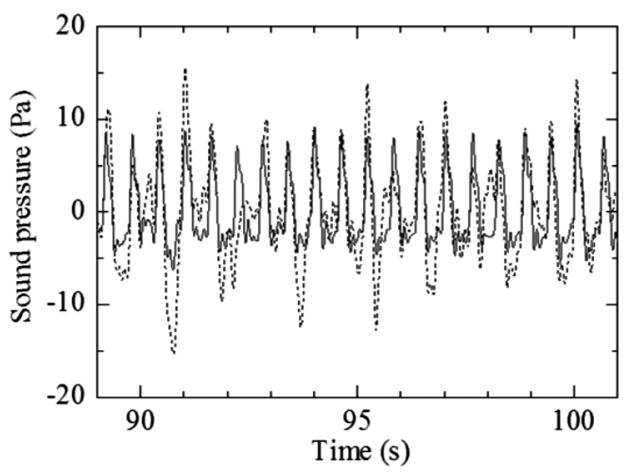

(f) Subject C
FIG. 5. Separated waveforms of the circulatory system (continuous line) and the respiratory system (dashed line) for the right ECP measured for three subjects [(a) subject A, (b) subject $\mathrm{B}$, and (c) subject $\mathrm{C}$ ] in a sitting position. The normal ICP waveform contains three phases: $P_{1}$ (percussion wave), representing the arterial pulsation; $P_{2}$ (tidal wave), representing the intracranial compliance; $P_{3}$ (dicrotic wave), representing the venous pulsation. Comparison of the sound pressure waveform characteristics among three subjects [(d) subject A, (e) subject B, and (f) subject $\mathrm{C}]$ for the right CAP (dashed line) and the right ECP (continuous line) taken during the supine period. 
follows the dicrotic notch on the down-slope of the individual ICP pulse waveform. Additional waves representing pulsations within the cerebral venous system may occur before the next $P_{1}$. Normally, the segments of the ICP pulse waveform progress in a descending staircase pattern, with $P_{1}$ as the highest waveform segment and $P_{3}$ as the lowest. As the ICP increases, cerebral compliance decreases, arterial pulses become more pronounced, and venous components disappear. The waveform acquired through the invasive technique shows the typical changes associated with an increase in ICP, with $P_{2}$ higher than $P_{1}$.

Therefore, the changes in the infrasonic emission waveform features of ECP in response to changes in ICP can be easily detected and can effectively discriminate between high and low SBP/ICP levels.

In healthy individuals, the forceful bounding of the carotid artery pulse during systole is palpable. The normal carotid artery pulsation has a single positive wave during systole, followed by the dicrotic notch (approximately timed to the second heart sound). The morphology of the carotid pulse signal reflects small volume changes within the artery with each heartbeat. The signal normally begins to rise abruptly with aortic ejection and reaches an initial peak (percussion wave) at the time of the maximum ejection force. A plateau or secondary wave (tidal wave) usually follows late in systole. This wave may primarily represent the reflected pulse wave returning from the upper body. Next, the wave falls smoothly to a point termed the dicrotic notch, which is produced by the abrupt closure of the aortic valve. In early diastole, there is often a small, positive wave, designated the dicrotic wave, which is most likely an effect of the pulse reflected from the lower body (Tavel, 1978). There is a slight delay between the cardiac apex impulse and the carotid artery pulse.

The sound pressure waveforms of the right CAPs (dashed line) from three supine subjects revealed time-varying characteristics, as shown in Figs. 5(d)-5(f). However, the sound pressure waveforms of the right ECPs (continuous line) for the same subjects were relatively stable

(i) The circulatory pulse waveforms mainly consist of three components: $P_{1}, P_{2}$, and $P_{3}$ as shown in Figs. 5(a), 5(b), and 5(c), respectively.

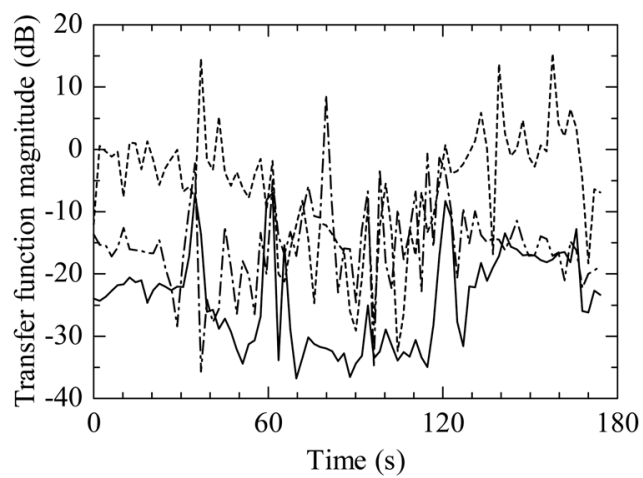

(a) Subject A

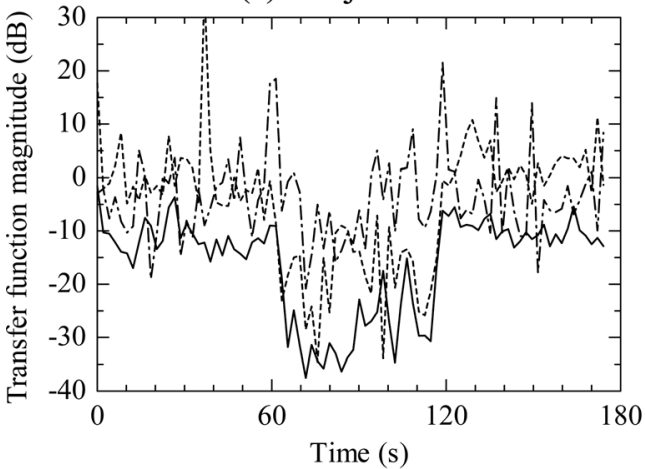

(b) Subject B

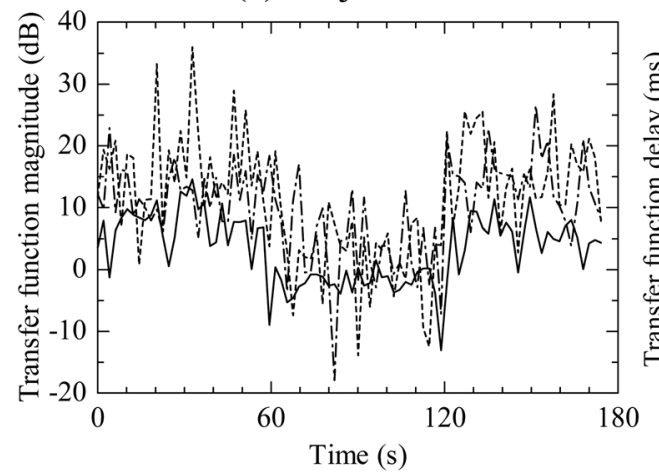

(c) Subject C

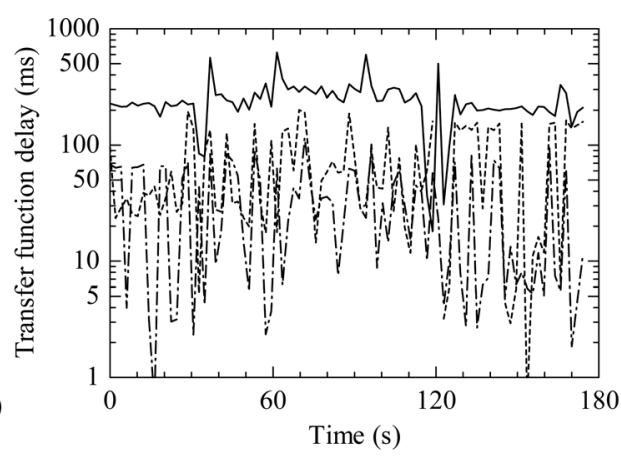

(d) Subject A

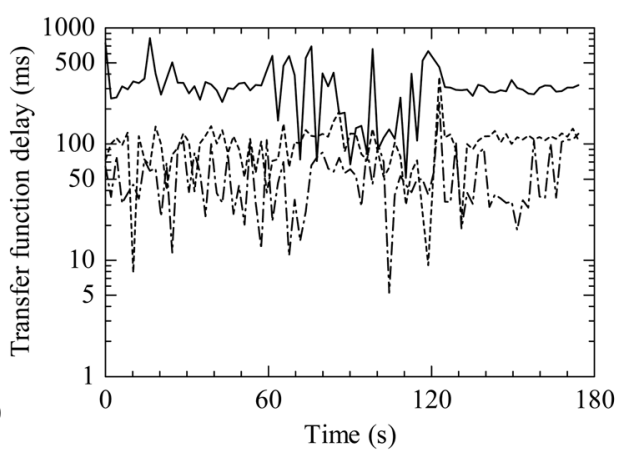

(e) Subject B

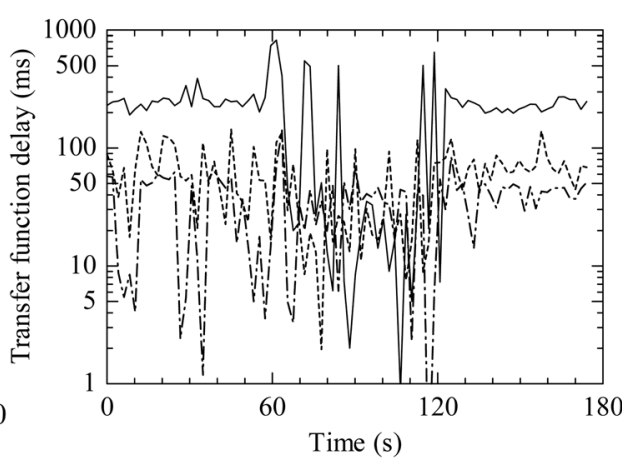

(f) Subject C
FIG. 6. The magnitude $(\mathrm{dB})$ of the transfer function is calculated in each $2.048 \mathrm{~s}$ window (Hanning window) for the three subjects [(a) subject A, (b) subject B, and (c) subject C]. Continuous line: fundamental frequency, dashed line: 4th harmonic, and long dashed singledotted line: 10th harmonic. The delay time (ms) of the transfer function is calculated in each $2.048 \mathrm{~s}$ window (Hanning window) for three subjects [(d) subject $A$, (e) subject $\mathrm{B}$, and (f) subject C]. Continuous line: fundamental frequency, dashed line: 4th harmonic, and long dashed single-dotted line: 10th harmonic. 
(ii) Under normal conditions, for Figs. 5(d) and 5(e), $P_{1}$ is the highest of the three waveform components.

(iii) For Fig. 5(f) with the highest SBP, $P_{2}$ is higher than $P_{1}$.

\section{Time-varying transfer function}

Typical CAP input and ECP output recordings at each SBP level are shown in Figs. 6(a)-6(c). The short-time Fourier transform analysis detected, one fundamental frequency and multiple harmonic components, and transfer functions were only evaluated at those discrete frequencies. Figures 6(a), 6(b), and 6(c) show the magnitude (dB) of the transfer function calculated for each time window (Hanning window), with windows separated by $2.048 \mathrm{~s}$ intervals, for the three subjects A, B, and C, respectively. In Fig. 6, the continuous line represents one fundamental frequency, the dashed line is the 4th harmonic component, and the dashed-dotted line represents the 10th harmonic component. The figures enable the following conclusions:

(i) The one-fundamental-frequency components (continuous lines) vary from $-35 \mathrm{~dB}$ to $10 \mathrm{~dB}$ depending on the three factors of time, postural position, and subject. The mean magnitude in the sitting position was approximately $-23 \mathrm{~dB}$ for subject $\mathrm{A},-11 \mathrm{~dB}$ for subject $B$, and $7 \mathrm{~dB}$ for subject $C$. The mean magnitude in the supine position was approximately $-35 \mathrm{~dB}$ for subject $\mathrm{A},-30 \mathrm{~dB}$ for subject $\mathrm{B}$, and $-2 \mathrm{~dB}$ for subject $\mathrm{C}$.

(ii) The 4th harmonic components (dashed lines) vary from $-33 \mathrm{~dB}$ to $35 \mathrm{~dB}$ depending on the three factors of time, postural position, and subject. The mean magnitude in the sitting position was approximately $-2 \mathrm{~dB}$ for subject $\mathrm{A}, 1 \mathrm{~dB}$ for subject $\mathrm{B}$, and $17 \mathrm{~dB}$ for subject $C$. The mean magnitude in the supine position was approximately $-20 \mathrm{~dB}$ for subject A, $-20 \mathrm{~dB}$ for subject $\mathrm{B}$, and $0 \mathrm{~dB}$ for subject $\mathrm{C}$.

(iii) The 10th harmonic components (dashed-dotted lines) vary from $-35 \mathrm{~dB}$ to $25 \mathrm{~dB}$ depending on the three factors of time, postural position, and subject. The mean magnitude in the sitting position was approximately $-18 \mathrm{~dB}$ for subject $\mathrm{A}, 5 \mathrm{~dB}$ for subject $\mathrm{B}$, and $12 \mathrm{~dB}$ for subject $\mathrm{C}$. The mean magnitude in the supine position was approximately $-18 \mathrm{~dB}$ for subject $\mathrm{A},-10 \mathrm{~dB}$ for subject $\mathrm{B}$, and $0 \mathrm{~dB}$ for subject $\mathrm{C}$.

The delay times (ms) of the transfer functions for the three subjects A, B, and C are shown in Figs. 6(d)-6(f), respectively. The continuous line represents one fundamental frequency, the dashed line is the 4th harmonic component, and the dashed-dotted line represents the 10th harmonic component. These figures enable the following conclusions:

(i) The one fundamental frequency components (continuous lines) vary from $1 \mathrm{~ms}$ to $800 \mathrm{~ms}$ depending on three factors of time, postural position, and subject. The mean delay time in the sitting position was approximately $210 \mathrm{~ms}$ for subject A, $250 \mathrm{~ms}$ for subject $\mathrm{B}$, and $220 \mathrm{~ms}$ for subject $\mathrm{C}$. The mean delay time in the supine position was approximately $300 \mathrm{~ms}$ for subject A, $200 \mathrm{~ms}$ for subject $\mathrm{B}$, and $30 \mathrm{~ms}$ for subject $\mathrm{C}$.

(ii) The 4th harmonic components (dashed lines) vary from $1 \mathrm{~ms}$ to $200 \mathrm{~ms}$ depending on the three factors of time, postural position, and subject. The mean delay time in the sitting position was approximately $40 \mathrm{~ms}$ for subject A, $100 \mathrm{~ms}$ for subject $\mathrm{B}$, and $60 \mathrm{~ms}$ for subject $\mathrm{C}$. The mean delay time in the supine position was approximately $50 \mathrm{~ms}$ for subject A, $100 \mathrm{~ms}$ for subject $\mathrm{B}$, and $25 \mathrm{~ms}$ for subject $\mathrm{C}$.

(iii) The 10th harmonic components (dashed-dotted lines) vary from $1 \mathrm{~ms}$ to $200 \mathrm{~ms}$ depending on the three factors of time, postural position, and subject. The mean delay time in the sitting position was approximately $20 \mathrm{~ms}$ for subject A, $40 \mathrm{~ms}$ for subject $\mathrm{B}$, and $35 \mathrm{~ms}$ for subject $\mathrm{C}$. The mean delay time in the supine position was approximately $30 \mathrm{~ms}$ for subject A, $50 \mathrm{~ms}$ for subject $\mathrm{B}$, and $40 \mathrm{~ms}$ for subject $\mathrm{C}$.

Therefore, the transfer functions were averaged over all sliding segments at one stationary point over time: the first seated interval was measured from $0 \mathrm{~s}$ to $54 \mathrm{~s}$; the supine interval from $71 \mathrm{~s}$ to $110 \mathrm{~s}$; and the second seated interval from $131 \mathrm{~s}$ to $176 \mathrm{~s}$. Figures 7(a)-7(d) represent the mean transfer functions calculated from the CAP input to ECP output data recorded during the first seated interval. Figure 7(a) represents the magnitude $(\mathrm{dB})$ transfer function as a function of the harmonic number: the transfer function for subject $\mathrm{A}$ $(\nabla)$ reaches a relative maximum magnitude $(2 \mathrm{~dB})$ at approximately the 5th harmonic component; $\mathrm{B}(\bigcirc)$ has two peaks $(1$ and $-2 \mathrm{~dB})$ at the 4 th and 7 th components; $\mathrm{C}(\triangle)$ also has two peaks $(18$ and $22 \mathrm{~dB})$ at the 4 th and 7 th components. Figure 7(b) shows that the standard deviations of the magnitude transfer function vary from $4 \mathrm{~dB}$ to $10 \mathrm{~dB}$. Figure 7(c) shows that the delay ( $\mathrm{ms}$ ) in the transfer function varies from $20 \mathrm{~ms}$ to $352 \mathrm{~ms}$. The mean delays of the first three harmonics were $278 \mathrm{~ms}, 131 \mathrm{~ms}$, and $100 \mathrm{~ms}$ (subject A: $231 \mathrm{~ms}, 122 \mathrm{~ms}$, and $92 \mathrm{~ms}$; subject B: $352 \mathrm{~ms}, 153 \mathrm{~ms}$, and $105 \mathrm{~ms}$; subject C: $251 \mathrm{~ms}, 118 \mathrm{~ms}$, and $102 \mathrm{~ms}$ ). Figure 7(d) shows that the standard deviations of the delay transfer functions vary from $20 \mathrm{~ms}$ to $140 \mathrm{~ms}$.

\section{DISCUSSION}

Noninvasive testing represents a powerful option for long-term care and follow-up. Physicians are able to identify changes in symptom, monitor disease status, and utilize their findings to identify appropriate candidates for neurosurgical intervention. Therefore, it is important to know the normal ranges for the magnitude and delay transfer function (from CAP to ECP) in healthy individuals. This investigation was initiated because the intracranial compliance may exhibit frequency dependence. This dependence may result from a particular mechanism within the cerebrovascular system such as filtering or convolution that treats different frequencies differently. Time-varying transfer function models are claimed to be capable of identifying inherent nonstationary dynamics in biomedical systems.

Every process in the body is controlled by signals sent from the brain, through the nerves, to each individual organ 

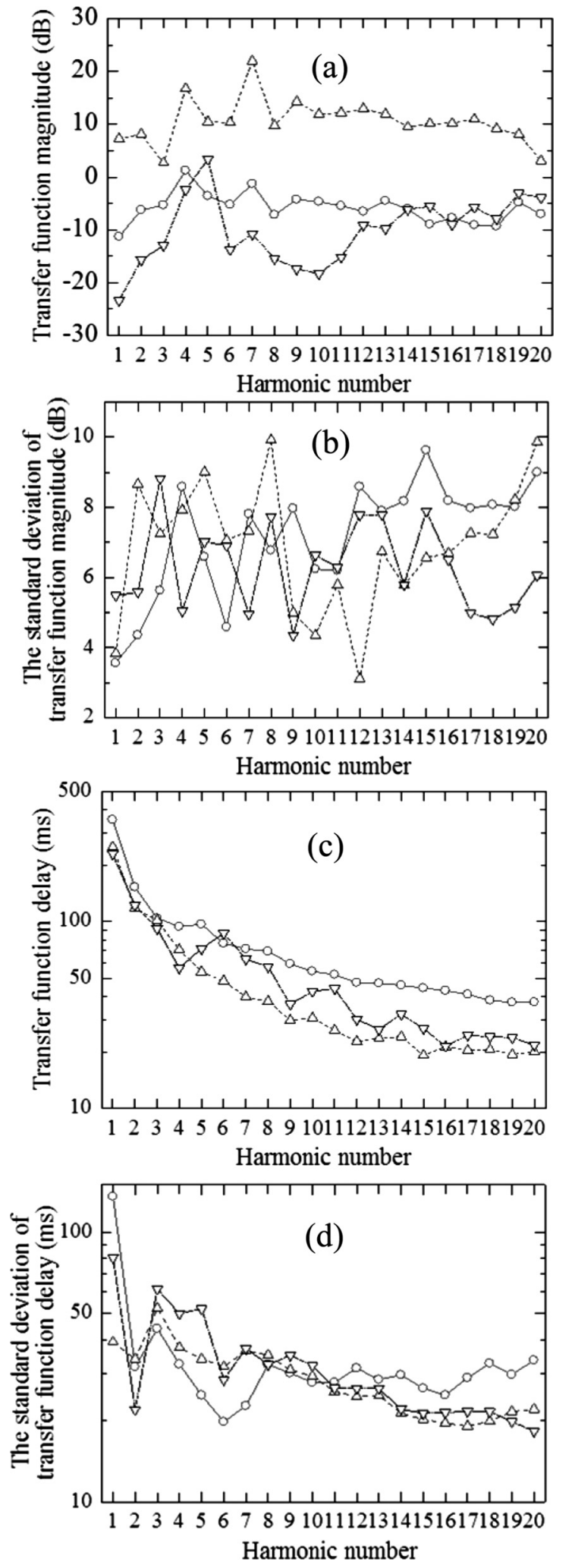

FIG. 7. Mean transfer functions from the CAP to ECP readings during the first seated interval. (a) Magnitude $(\mathrm{dB})$ as a function of the harmonic number. $\nabla$ : subject $A, \bigcirc$ : subject $B$, and $\triangle$ : subject $C$. (b) Standard deviation of magnitude $(\mathrm{dB}),(\mathrm{c})$ delay $(\mathrm{ms})$, and (d) standard deviation of delay (ms).

or muscle. These background tasks are referred to as the "autonomic nervous system." Heart rate, sleep, digestion, and vascular pressure are among the normal bodily functions that do not require conscious intervention. An increasing amount of neuroimaging evidence supports the hypothesis that chronic fatigue syndrome patients have structural or functional abnormalities within the brain. The syndrome is characterized by a wide variety of symptoms such as fatigue, weakness, muscle and joint pain, headache, memory and concentration difficulties, and difficulty sleeping.

Tables V and VI show which parameters load strongly on the factors produced by the PCA.

\section{A. Relationships between the magnitude of the transfer function and response factors}

Figures 8(a)-8(d) show the normal range of the magnitude of the transfer function, as a function of the harmonic number of the transfer function from the right CAP to the right ECP during the first seated interval. The continuous lines and the dashed-double-dotted lines demarcate the \pm 1 standard deviations from the mean (averaged over 419 data points). Both frequency transfer functions are basic highpass filter functions, and the differences between +1 and -1 the standard deviations from the mean are approximately $16 \mathrm{~dB}$. Below the cutoff frequency at the 4th harmonic component, the attenuation increases at a rate of approximately $6 \mathrm{~dB}$ per octave. These response curves are affected by the stethoscope response [see Fig. 2(f)].

Table $\mathrm{V}$ shows the relationships between the dependent variables (the harmonic numbers selected by the PCA) and a set of six independent parameters (SBP, SAA, pulse rate, age, sleeping time, and accumulated fatigue); the regression coefficients from multiple regressions for the measured parameters, the constants, and the correlation coefficients. The independent parameters in the model are not correlated with one another (correlation coefficients of 0.2 or less). Table V(a) shows the magnitude of the transfer function from CAP to ECP. Table V(b) shows the standard deviations of the magnitude of the transfer function. The correlation coefficient is an indicator of how well the model fits the data as shown in Table V. Table V(a) shows that (i) the second harmonic of the magnitude transfer function (Pearson $r=0.443)$ was significantly affected by SBP $(p<0.001)$, SAA $(p<0.001)$, pulse rate $(p<0.001)$, and accumulated fatigue $(p<0.05)$, (ii) the ninth harmonic (Pearson $r=0.496$ ) was significantly affected by SBP $(p<0.001)$, SAA $(p<0.001)$, and pulse rate $(p<0.001)$, and (iii) the sixteenth harmonic (Pearson $r=0.566)$ was significantly affected by SBP $(p<0.001)$, SAA $(p<0.001)$, age $(p<0.001)$, sleeping time $(p<0.001)$, and accumulated fatigue $(p<0.001)$. Table $\mathrm{V}(\mathrm{b})$ shows that (i) the first harmonic of the standard deviation of the magnitude transfer function (Pearson $r=0.320$ ) was significantly affected by accumulated fatigue $(p<0.001)$, gender $(p<0.01)$, and SAA $(p<0.01)$, and (ii) the second harmonic (Pearson $r=0.227$ ) was significantly affected by accumulated fatigue $(p<0.001)$, age $(p<0.01)$, and gender $(p<0.05)$.

\section{Systolic blood pressure (SBP)}

The SBP values were within the range of 90-239 $\mathrm{mmHg}$. Figure 8(a) demonstrates the following: (i) the mean magnitude of the transfer function $(\triangle)$, which is in the range of 161-239 mmHg (using 12 data points), falls above the upper 
TABLE V. The relationships between the dependent variables (the harmonic numbers selected by the PCA) and a set of 6 independent parameters. The regression coefficients for multiple regressions of the measured parameters and the corresponding correlation coefficients. (a) The magnitude of the transfer function from CAP to ECP, and (b) the standard deviation of the magnitude of the transfer function. $* p<0.05$, ** $p<0.01$, *** $p<0.001$, NS: not significant.

(a) The magnitude of the transfer function

\begin{tabular}{|c|c|c|c|c|c|c|c|c|}
\hline Harmonic number & $\begin{array}{c}\text { Systolic } \\
\text { blood pressure }\end{array}$ & $\begin{array}{c}\text { Salivary amylase } \\
\text { activity }\end{array}$ & Pulse rate & Age & Sleeping time & $\begin{array}{l}\text { Accumulated } \\
\text { fatigue }\end{array}$ & Constant & $\begin{array}{l}\text { Correlation } \\
\text { coefficient }\end{array}$ \\
\hline 2nd & $0.178 * * *$ & $0.028 * * *$ & $-0.178 * * *$ & NS & NS & $0.125^{*}$ & -27.309 & 0.443 \\
\hline 9 th & $0.195 * * *$ & $0.019 * * *$ & $-0.123 * * *$ & NS & NS & NS & -26.535 & 0.496 \\
\hline 16th & $0.083 * * *$ & $0.023 * * *$ & NS & $0.242 * * *$ & $-1.158 * * *$ & $-2.589 * * *$ & -20.371 & 0.566 \\
\hline
\end{tabular}

(b) The standard deviation of the magnitude of the transfer function

\begin{tabular}{lccccc}
\hline Harmonic number & Accumulated fatigue & Age & Gender & $\begin{array}{c}\text { Salivary amylase } \\
\text { activity }\end{array}$ & $\begin{array}{c}\text { Correlation } \\
\text { coefficient }\end{array}$ \\
\hline 1st & $0.251^{* * *}$ & $\mathrm{NS}$ & $-0.588^{* *}$ & $0.004 * *$ & 4.409 \\
2nd & $0.240^{* * *}$ & $0.029^{* *}$ & $-0.373^{*}$ & & 0.320 \\
7 th & $0.070^{\mathrm{NS}}$ & $-0.015^{\mathrm{NS}}$ & $-0.385^{\mathrm{NS}}$ & & 5.714 \\
\hline \hline
\end{tabular}

limit curve (the mean plus one standard deviation); (ii) the three mean magnitudes of transfer functions $(\boldsymbol{\Delta}, \bigcirc$, and $\triangle$ ), which fall in the ranges of $90-106 \mathrm{mmHg}$ (42 data points), $107-143 \mathrm{mmHg}$ (340 data points), and 144-160 mmHg (25 data points), fall between the upper and lower limits (demarcated by the mean \pm 1 standard deviation).

\section{Salivary amylase activity (SAA)}

Normalized SAA was correlated with the mental and physical fatigue states of the study participants, supporting the idea that salivary amylase activity might be a good index for psychological research (Yamaguchi et al., 2006). The SAA values of the participants fell within the range of 10 to $200 \mathrm{kU} / \mathrm{l}$. Figure 8(b) shows the mean magnitudes as a function of the harmonic number of the transfer function from the right CAP to the right ECP during the first seated interval, classified by SAA (10-49 kU/1, 50-100 kU/1, and 101$200 \mathrm{kU} / \mathrm{l})$. This figure shows that the mean magnitude curves $(\bigcirc$ and $\nabla$ ) that are associated with a range of SAA between 10 and $100 \mathrm{kU} / \mathrm{l}$ are well aligned with the central points between the upper and lower limits (demarcated by the mean \pm 1 standard deviation). By comparison, the mean magnitude curve $(\triangle)$ that is associated with a higher range of SAA between 101 and $200 \mathrm{kU} / \mathrm{l}$ is aligned with approximately $75 \%$ of the central points between the upper and lower limits.

\section{Age}

Age is the greatest risk factor for cardiac or respiratory failure. Figure 8(c) shows the mean magnitudes as a function of the harmonic number of the transfer function from the right CAP to the right ECP during the first seated interval, classified by age (19-30 years, 31-49 years, and 50-57 years). This figure shows that between the 12 th to 20 th harmonic numbers, the difference between the magnitudes of the 19-30-year curve $(\nabla)$ and the 31-57-year curves $(\bigcirc$ and $\triangle$ ) is approximately $6 \mathrm{~dB}$.

\section{Sleeping time}

Quality sleep can eliminate tiredness and strengthen immunity. However, good sleep is increasingly becoming a

TABLE VI. The relationships between the dependents (the harmonic numbers selected by the PCA) and a set of 5 independent parameters. The regression coefficients for multiple regressions of the measured parameters and the corresponding correlation coefficients. (a) The time delay transfer function from CAP to ECP, and (b) the standard deviations of the time delay transfer function. $* p<0.05, * * p<0.01$, *** $p<0.001$, NS: not significant.

(a) The time delay transfer function

\begin{tabular}{|c|c|c|c|c|c|c|c|}
\hline Harmonic number & Pulse rate & Gender & Sleeping time & Age & $\begin{array}{c}\text { Systolic } \\
\text { blood pressure }\end{array}$ & Constant & $\begin{array}{l}\text { Correlation } \\
\text { coefficient }\end{array}$ \\
\hline $1 \mathrm{st}$ & $-1.820 * * *$ & $-36.996 * * *$ & $-9.115^{*}$ & NS & NS & 528.798 & 0.333 \\
\hline 5 th & $-0.571 * * *$ & NS & NS & $0.219 * * *$ & NS & 108.114 & 0.428 \\
\hline 16 th & $-0.301 * * *$ & NS & NS & NS & $-0.064^{*}$ & 61.228 & 0.342 \\
\hline
\end{tabular}

(b) The standard deviation of the time delay transfer function

\begin{tabular}{|c|c|c|c|c|c|c|c|}
\hline Harmonic number & Salivary amylase activity & Accumulated fatigue & Gender & Age & Pulse rate & Constant & $\begin{array}{l}\text { Correlation } \\
\text { coefficient }\end{array}$ \\
\hline $1 \mathrm{st}$ & $0.270 * * *$ & $13.647 * * *$ & NS & NS & NS & 82.578 & 0.383 \\
\hline 2nd & $0.070 * * *$ & NS & NS & $0.599 * * *$ & $-0.923 * * *$ & 111.022 & 0.426 \\
\hline 8th & NS & NS & $-4.163 * * *$ & $0.239 * * *$ & $-0.272 * * *$ & 45.493 & 0.355 \\
\hline 17 th & $0.016^{* *}$ & NS & NS & $0.083 *$ & $-0.228 * * *$ & 40.755 & 0.385 \\
\hline
\end{tabular}


luxury in the modern age. People who suffer from sleep deprivation are more susceptible to developing a poorly responsive immune system and more likely to suffer from restlessness, anxiety, poor memory, high blood pressure, and cardiovascular disease. Figure 8(d) shows the mean magnitudes, as a function of the harmonic number of the transfer function from the right CAP to the right ECP during the first seated interval, classified by the amount of sleeping time. From this figure, the following can be observed: (i) the mean magnitude curve (white circles) of the transfer function with an average amount of sleep, from $5 \mathrm{~h} 18 \mathrm{~min}$ to $7 \mathrm{~h} 25 \mathrm{~min}$ (300 data points), is well aligned with the central points between the upper and lower limits (demarcated by the mean \pm 1 standard deviation); (ii) the mean magnitude curve $(\triangle$ ) of the transfer function with a short amount of sleep, from $3 \mathrm{~h} 30 \mathrm{~min}$ to $5 \mathrm{~h} 17 \mathrm{~min}$ (75 data points), is aligned with approximately $65 \%$ of the central points between the upper and lower limits; (iii) the mean magnitude curve $(\nabla)$ of the transfer function with a long amount of sleep, from $7 \mathrm{~h}$ $26 \mathrm{~min}$ to $10 \mathrm{~h}$ (44 data points), are aligned with approximately $35 \%$ points of the central points between the upper and lower limits.

\section{Postural change}

Short-term cardiovascular responses to postural shifts from a sitting position to a supine position involve complex interactions between the autonomic nervous system, which regulates blood pressure, and cerebral autoregulation, which maintains cerebral perfusion. Figure 9(a) shows the mean magnitude curves, as a function of the harmonic number of the transfer function from the right CAP to the right ECP during the first seated interval, the supine interval, and the second seated interval. This figure demonstrates the following: (i) the mean magnitude curve ( $)$ of the transfer function from the second seated interval is well aligned with the curve $(\bigcirc)$ from the first sitting position; (ii) the mean magnitude curve ( $\square$ ) from the supine interval attenuates from $2 \mathrm{~dB}$ to $10 \mathrm{~dB}$, relative to the curves from the seated intervals.

\section{Accumulated fatigue}

A scale from the checklist for the accumulation of general fatigue in workers was used to measure psychosomatic symptoms. The overall assessment determined the degree of work-related burdens on a scale from 0 to 7 and was based on a subjective evaluation of symptoms and work conditions. The relationship between the numerical scaling system ( 0 to 7) and the degree of work-related burden (low, rather high, high, and very high) is as follows: 0 and 1: low; 2 and 3: rather high; 4 and 5: high; 6 and 7: very high. This checklist is an evidence-based instrument that can be used to assess the risk for health impairments secondary to work-related burdens. Any score from 2 to 7 indicates a risk for the accumulation of fatigue and may indicate a need to improve specific work conditions. Randomization tests can be used if the data have a serial dependency, and these tests can be applied to various single-case designs (Edington, 1975).
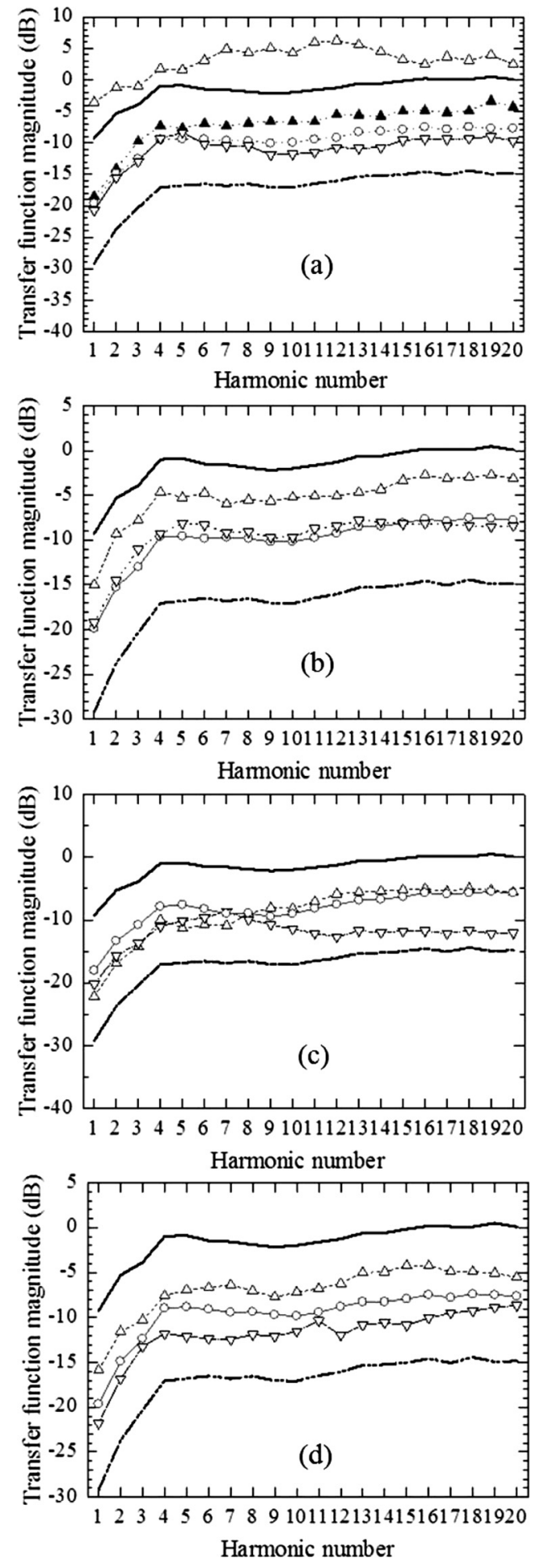

FIG. 8. The magnitudes $(\mathrm{dB})$ as a function of the harmonic number of the transfer function from the right CAP to the right ECP during the first seated interval. The continuous and dashed-double-dotted lines demarcate \pm 1 standard deviation from the mean based on 419 data points. (a) $\triangle$ : systolic blood pressure between 161-239 mmHg (12 data points); $\mathbf{\Delta}$ : systolic blood pressure between $144-160 \mathrm{mmHg}$ (25 data points); $\bigcirc$ : systolic blood pressure between $107-143 \mathrm{mmHg}$ (340 data points); and $\nabla$ : systolic blood pressure between $90-106 \mathrm{mmHg}$ (42 data points). (b) $\triangle$ : salivary amylase between 101-200 kU/l (97 data points); $\bigcirc$ : salivary amylase between 50$100 \mathrm{kU} / \mathrm{l}$ (121 data points); $\nabla$ : salivary amylase between $10-49 \mathrm{kU} / \mathrm{l}$ (199 data points). (c) $\triangle: 50-57$ years of age (63 data points); $\bigcirc: 31-49$ years of age (245 data points); $\nabla: 19-30$ years of age (111 data points). (d) $\triangle$ : sleeping time from $3 \mathrm{~h} 30 \mathrm{~min}$ to $5 \mathrm{~h} 17 \mathrm{~min}$ (75 data points); $\bigcirc$ : sleeping time from $5 \mathrm{~h} 18 \mathrm{~min}$ to $7 \mathrm{~h} 25 \mathrm{~min}$ (300 data points); $\nabla$ : sleeping time from $7 \mathrm{~h}$ $26 \mathrm{~min}$ to $10 \mathrm{~h}$ (44 data points). 
The standard deviations of the magnitudes, as a function of the harmonic number of the transfer function from the right CAP to the right ECP during the first seated interval, were classified according to the scale points assigned to the work-related burdens, as shown in Fig. 9(b). The continuous line and the dashed-double-dotted line demarcate \pm 1 standard deviation of the mean determined from 397 data points. This figure shows: (i) as the degree of the work-related burden increases from 0 to 5 , the standard deviation increases by $1 \mathrm{~dB}$; (ii) the results of randomization tests reveal the following: the one-sided probability of the one fundamental component is $p=0.00038$; the 2nd harmonic component: $p=0.003$; the 3rd harmonic component: $p=0.0797$; the 4 th harmonic component: $p=0.0184$; and the 5th harmonic component: $p=0.011$; (iii) if the desired significance level for a result is 0.05 , only the 3 rd harmonic component fails to achieve the 0.05 level of significance, although it is significant at the 0.1 level.
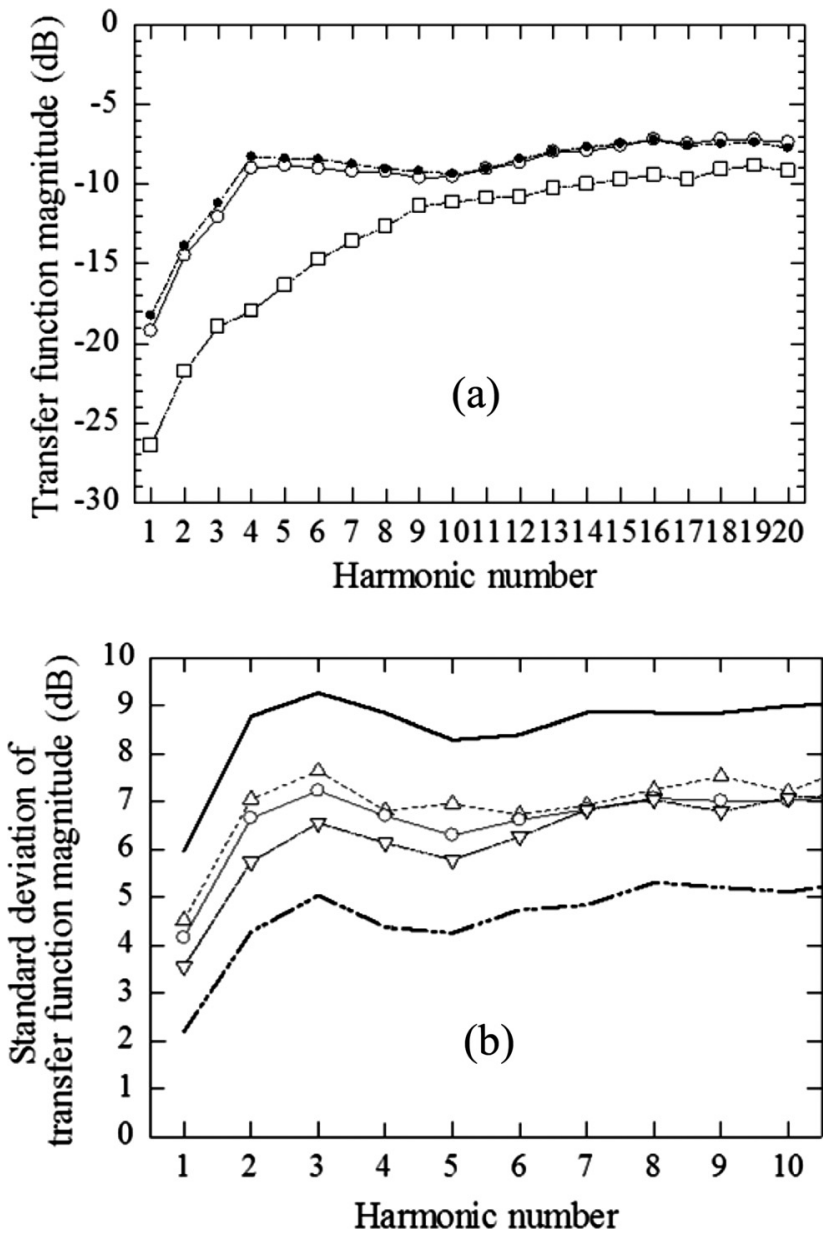

FIG. 9. (a) Magnitudes (dB) as a function of the harmonic number of the transfer function from the right CAP to the right $\mathrm{ECP}$ during shifts in posture. $\bigcirc$ : the first seated interval; $\bigcirc$ : the second seated interval; $\square:$ the supine interval. (b) Standard deviation of the transfer function magnitude $(\mathrm{dB})$ as a function of the harmonic number of the transfer function from the right CAP to the right ECP during the first seated interval. The continuous and dasheddouble-dotted lines demarcate \pm 1 standard deviation from the mean on 397 data points. We used a scale from the checklist for the accumulation of general fatigue of workers to measure psychosomatic symptoms. $\triangle$ : the psychosomatic symptoms range from 4 to 5 (52 data points); $\bigcirc$ : the psychosomatic symptoms range from 2 to 3 (52 data points); $\nabla$ : the psychosomatic symptoms range from 0 to 1 (293 data points).

\section{B. Relationships between the time delay transfer function and response factors}

Figures 10 (a) and 10(c) show the normal range for the time delay as a function of the harmonic number of the transfer function from the right CAP to the right ECP during the first seated interval. The continuous line and the dashed-double-dotted line demarcate \pm 1 standard deviation from the mean of 418 data points. This figure indicates: (i) the time delay at the one fundamental frequency normally ranges from $193 \mathrm{~ms}$ to $381 \mathrm{~ms}$; (ii) the range of the 5 th harmonic component is from $62 \mathrm{~ms}$ to $93 \mathrm{~ms}$; (iii) the $10 \mathrm{th}$ : $32 \mathrm{~ms}$ to $56 \mathrm{~ms}$; and (iv) the 20th: $16 \mathrm{~ms}$ to $36 \mathrm{~ms}$.

Table VI shows the relationships between the dependents (the harmonic numbers selected by the PCA) and a set of seven independent parameters (pulse rate, gender, sleeping time, age, SBP, SAA, and accumulated fatigue), the regression coefficients for multiple regressions of the measured parameters, the constants, and the correlation coefficients. The independent parameters in the model are not correlated with one another, with correlation coefficients of 0.2 or less. Table V1(a) shows the time delay transfer function from CAP to ECP. Table V1(b) shows the standard deviations of the time delay transfer functions. The correlation coefficient is an indicator of how well the model fits the data as shown in Table VI. Table V1(a) shows that (i) the first harmonic of the time delay transfer function (Pearson $r=0.333$ ) was significantly affected by pulse rate $(p<0.001)$, gender $(p<0.001)$, and sleeping time $(p<0.05)$; (ii) the fifth harmonic $(r=0.428)$ was significantly affected by pulse rate $(p<0.001)$ and age $(p<0.001)$; and (iii) the sixteenth harmonic $(r=0.342)$ was significantly affected by pulse rate $(p<0.001)$ and SBP $(p<0.05)$. Table V(b) shows that (i) the first harmonic of the standard deviations of the time delay transfer function $(r=0.383)$ was significantly affected by SAA $(p<0.001)$ and accumulated fatigue $(p<0.001)$; (ii) the second harmonic $(r=0.426)$ was significantly affected by SAA $(p<0.001)$, age $(p<0.001)$, and pulse rate $(p<0.001)$; (iii) the eighth harmonic $(r=0.355)$ was significantly affected by gender $(p<0.001)$, age $(p<0.001)$, and pulse rate $(p<0.001)$; and (iv) the seventeenth harmonic $(r=0.385)$ was significantly affected by SAA $(p<0.01)$, age $(p<0.05)$, and pulse rate $(p<0.001)$.

\section{Pulse rate}

The measured pulse rates were within the range of 45104 beats/minute. From Fig. 10(a), the three mean delay transfer functions $(\triangle, \bigcirc$, and $\nabla)$ decrease from the upper to lower limits (demarcated by the mean \pm 1 standard deviations), ranging from 45-61 beats/minute (64 data points), $62-83$ beats/minute (282 data points), and 84-104 beats/minute (72 data points), respectively.

Figure 10(b) shows the standard deviations of the delay times as a function of the harmonic number of the transfer function from the right CAP to the right ECP during the first seated interval. The continuous line and the dashed-doubledotted line demarcate \pm 1 standard deviation from the mean of 418 data points. The figure shows that the three mean standard deviations of the time delay transfer functions $(\triangle$, $\bigcirc$, and $\nabla$ ) decrease from the upper to lower limits. 

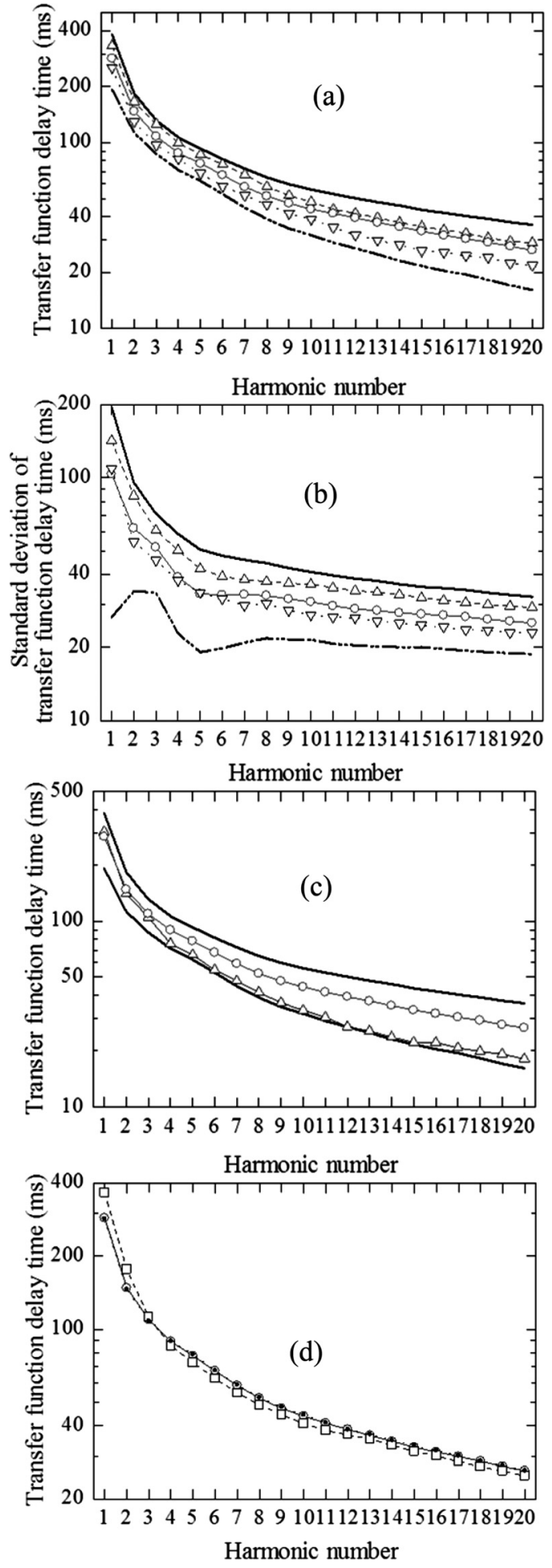

FIG. 10. The time delay (ms) as a function of the harmonic number of the transfer function from the right CAP to the right ECP during the first seated interval. In panels (a), (b), and (c) the continuous and dashed-double-dotted lines demarcate \pm 1 standard deviation from the mean based on 418 data points. (a) $\triangle$ : pulse rates from 45 to 61 beats/minute (64 data points); $\bigcirc$ : pulse rates from 62 to 83 beats/minute ( 282 data points); $\nabla$ : pulse rates from 84 to 104 beats/minute ( 72 data points). (b) Standard deviation of the delay time (ms) as a function of the harmonic number of the transfer function from the right CAP to the right ECP during the first seated interval. Symbols are the same as in (a). (c) $\triangle$ : systolic blood pressure between 161$239 \mathrm{mmHg}$ (12 data points); $\bigcirc$ : systolic blood pressure between $107-$ $143 \mathrm{mmHg}$ (340 data points). (d) Mean delay time (ms) as a function of the harmonic number of the transfer function from the right CAP to the right ECP readings. $\bigcirc$ : the first seated period; $\bigcirc$ : the second seated period; and $\square$ : the supine period.

\section{2. $S B P$}

Figure 10(c) presents a comparison between the normal range $(107-143 \mathrm{mmHg})$ and the highest range (161$239 \mathrm{mmHg}$ ) of SBP. The following can be observed based on the figure: (i) the normal range coincides with $286 \mathrm{~ms}$, and the highest range coincides with $304 \mathrm{~ms}$ at one fundamental frequency; (ii) the normal range coincides with $149 \mathrm{~ms}$, and the highest range coincides with $141 \mathrm{~ms}$ at the 2nd harmonic component; (iii) the highest range curve $(\triangle)$ aligns closely with the lower limit curve (dashed-double-dotted line) between the 4th and 20th harmonics.

\section{Postural change}

Figure 10(d) shows the mean delay times (ms) as a function of the harmonic number of the transfer function from the right $\mathrm{CAP}$ to the right $\mathrm{ECP}$ readings taken during changes in posture. The following can be observed based on the figure: (i) the mean delay time curve (white circles) from the first seated interval is closely aligned with the curve from the second seated interval (@); (ii) at one fundamental frequency, the mean delay time from the first seated interval is $287 \mathrm{~ms}$, and the mean delay time of the supine interval is $365 \mathrm{~ms}$; (iii) at the 2nd harmonic component, the mean delay time from the first seated interval is $148 \mathrm{~ms}$, and the mean delay time from the supine interval is $176 \mathrm{~ms}$; (iv) however, between the 4th and 20th harmonic components, the mean delay times for the first seated interval are longer than those for the supine interval, by $1 \mathrm{~ms}$ to $5 \mathrm{~ms}$.

\section{Discriminant analysis}

Figure 8 shows that only the high SBP leads to a significant elevation in the magnitude of the transfer function. Other study variables do not cause significant changes in the magnitude of the transfer function, with effects within standard deviations. Therefore, a discriminant analysis was used to classify cases based on the values of the transfer function dependent. If this discriminant analysis is effective for a set of data, then the classification table of three groups' estimates will yield a high percentage of correct predictions. Three groups were defined: group I was characterized by transfer function dependents with values more than one standard deviation above the mean (the magnitude, the standard deviation of the magnitude, the time delay, and the standard deviation of the time delay), group II by approximately $68 \%$ of the data within one standard deviation (higher or lower) from the mean, and group III by values more than one standard deviation below the mean. A discriminant analysis had two steps: (1) an $F$ test (Wilks' lambda) was used to test whether the discriminant model as a whole was significant; and (2) if the $F$ test showed significance, then the individual independent variables were assessed to identify those that differed significantly, and these were used to classify the dependent variable.

From the results of discriminant analysis, (i) for the magnitude of the second harmonic number of the transfer function, SBP, SAA, pulse rate, and accumulated fatigue were significant predictors $[F(3,20)=8.08, p<0.001$, canonical 
correlation $=0.428$, Wilkes' $\lambda=0.81$, and $44.1 \%$ of original grouped cases correctly classified]; (ii) for magnitude of the ninth harmonic number of the transfer function, SBP, SAA, and pulse rate were significant predictors $[F(3,12)=13.12$, $p<0.001$, canonical correlation $=0.458$, Wilkes' $\lambda=0.78$, and $47.9 \%$ of original grouped cases correctly classified]; (iii) for magnitude of the sixteenth harmonic number of the transfer function, SBP, SAA, age, sleeping time, and accumulated fatigue were significant predictors $[F(3,30)=8.76, p<0.001$, canonical correlation $=0.534$, Wilkes' $\lambda=0.69$, and $59.9 \%$ of original grouped cases correctly classified]; (iv) for the standard deviation of the magnitude of the first harmonic number of the transfer function, accumulated fatigue, gender, and SAA were significant predictors $[F(3,20)=4.51, p<0.001$, canonical correlation $=0.311$, Wilkes' $\lambda=0.89$, and $43.8 \%$ of original grouped cases correctly classified]; (v) for the time delay of the first harmonic number of the transfer function, pulse rate, gender, and sleeping time were significant predictors $[F(3,12)=1.65, p<0.072$, canonical correlation $=0.301$, Wilkes' $\lambda=0.90$, and $35.0 \%$ of original grouped cases correctly classified]; (vi) for the time delay of the fifth harmonic number of the transfer function, pulse rate, and age were significant predictors $[F(3,6)=5.11, p<0.001$, canonical correlation $=0.404$, Wilkes' $\lambda=0.83$, and $47.6 \%$ of original grouped cases correctly classified]; and (vii) for the standard deviation of the time delay of the second harmonic number of the transfer function, amylase, age, and pulse rate were significant predictors $[F(2,6)=1.92, p<0.073$, canonical correlation $=0.104$, Wilkes' $\lambda=0.99$, and $58.4 \%$ of original grouped cases correctly classified].

What physiological mechanism could cause these variables to affect the measured transfer function? The data in the present study show that for the measured transfer function, an average of $48.1 \%$ of cases were correctly classified into the original three groups by the following significant predictors: SBP, SAA, pulse rate, accumulated fatigue, age, gender, and sleeping time. However, many other factors can be considered including physiological factors, changes in the mechanical properties of cells, and so forth. Full appreciation and clinical application of this phenomenon will require integration of the hydrostatic, hemodynamic, and perhaps biomechanical aspects of the brain.

\section{CONCLUSIONS}

In this study, we have proposed to treat the infrasound pressure of the carotid artery as an input that is composed of multiple frequencies and that is represented in the output infrasonic emissions from the TM according to the transfer function imposed by the intracranial system on the carotid input signal. Transfer functions in 40 normal workers (aged 19 to 57 years old) were characterized, involving the analysis of 446 data points.

The results have demonstrated the following:

The ECP waveform produces a pulsatile signal with a slow respiratory component that is superimposed on a biphasic signal that is synchronous with the cardiac cycle; the mean SPL of the right ECP for the circula- tory system during a seated interval is $98.4 \mathrm{~dB}$; the mean SPL for the respiratory system is $83.5 \mathrm{~dB}$.

(ii) The respiratory ECP waveform is correlated with the instantaneous heart rate, with a mean maximum cross-correlation coefficient of approximately 0.35 .

(iii) Changes in the infrasonic emission waveform features of ECP can be clearly observed, and they allow for the discrimination between high and low SBP (ICP) levels; the correlation coefficient between the average root mean square sound pressure $(\mathrm{Pa})$ of the right $\mathrm{ECP}$ and the average SBP (mmHg) during the first and second seated intervals is 0.581 (significant at the 0.01 level).

(iv) The normal range of transfer function magnitudes (CAP to ECP) in healthy people represents a basic high-pass filter, and the range covered by +1 to -1 standard deviation from the mean is approximately $16 \mathrm{~dB}$. Below the cutoff frequency at the 4th harmonic component, the attenuation increases at a rate of approximately $6 \mathrm{~dB}$ per octave.

(v) Various fatigue-related risk factors that affect the mean magnitude and delay transfer functions between CAP to ECP among the general workers studied here systolic blood pressure, salivary amylase activity, age, sleeping time, postural changes, chronic fatigue, and pulse rate.

Therefore, noninvasive measurement of the transfer function using infrasonic emissions from the TM and the carotid artery may become a useful tool to improve the quality of health care for general workers.

In the future, noninvasive testing will represent a powerful option for long-term and follow-up care. Physicians will be able to detect symptom changes, monitor disease status, and utilize findings to identify appropriate candidates for neurosurgical interventions based on the normal ranges of the magnitude and delay transfer functions (CAP to ECP) in healthy people. This method does not allow us to measure absolute ICP levels, but they may be predicted from the brain elastance index (change in pressure related ECP per unit change in volume related CAP).

\section{ACKNOWLEDGMENTS}

This research was supported by the Adaptable and Seamless Technology Transfer Program through Targetdriven R\&D (A-STEP) (Grant No. 231Z01795) support from Japan Science and Technology (JST), and also by a Grantin-Aid for Scientific Research (C) (Grant No. 23500257) from the Japan Society for the Promotion of Science (JSPS).

Abella, M., Formolo, J., and Penney, D. G. (1992). "Comparison of the acoustic properties of six popular stethoscopes," J. Acoust. Soc. Am. 91, 2224-2228.

Chopp, M., and Portnoy, H. D. (1980). "System analysis of intracranial pressure. Comparison with volume-pressure test and CSF-pulse amplitude analysis," J. Neurosurg. 53, 516-527.

Ciuman, R. R. (2009). "Communication routes between intracranial spaces and inner ear: Function, pathophysiologic importance and relations with inner ear diseases," Am. J. Otolaryngol. 30, 193-202.

Czosnyka, M., Smielewski, P., Timofeev, I., Lavinio, A., Guazzo, E., Hutchinson, P., and Pickard, J. D. (2007). "Intracranial pressure: More than a number," J. Neurosurgical Focus 22, 1-7. 
Edington, E. S. (1975). "Randomization tests for one-subject operant experiments," J. Psychol. 90, 57-68.

Field, A. (2010). Third Edition of Discovering Statistics Using SPSS (SAGE Publications, London), Chap. 4.2, pp. 116-161, and Chap. 11, pp. 423-469. Japan Industrial Safety and Health Association, Tokyo: JISHA. (2002). "The checklist for the accumulation of general fatigue of the workers," http://www.mhlw.go.jp/houdou/2003/05/h0520-3.html (Last accessed 0207-2012) (in Japanese).

Kirkness, C. J., Mitchell, P. H., Burr, R. L., March, K. S., and Newell, D. W. (2000). "Intracranial pressure waveform analysis: Clinical and research implications," J. Neurosci. Nurs. 32, 271-277.

Koidan, W. (1954). "An acoustic method for the measurement of vibration amplitudes," J. Acoust. Soc. Am. 26, 428-433.

Lundberg, N. (1960). "Continuous recording and control of ventricular fluid pressure in neurosurgical practice," Acta. Psychiatr. Scand. Suppl. 36, 1-193.

March, K. (2000). "Intracranial pressure monitoring and assessing intracranial compliance in brain injury," Crit. Care. Nurs. Clin. North Am. 12, 429-436.

Marchbanks, R. J., Reid, A., Martin, A. M., Brightwell, A. P., and Bateman, D. (1987). "The effect of raised intracranial pressure on intracochlear fluid pressure: Three case studies,” Br. J. Audiol. 21, 127-130.

Matsubara, S., Furihata, K., and Yanagisawa, T. (1999). "Electroacoustic transducers of earplug type for active noise control in external auditory canal," in ACTIVE 99, December 4-6, Fort Lauderdale, FL, pp. 1257-1266.

Matsuyama, M. (1995). "Recording of the effects on the external auditory canal pressure waves of intracranial pressure pulse waves and blood pressure waves," Keio J. Med. 72, 497-509 (in Japanese).

Paulat, K., Brucher, R., and Russell, D. (1999). "Non-invasive assessment of intracranial Compliance," Cerebrovasc. Dis. 9(Suppl 2), 1-55.

Piper, I. R., Chan, K. H., Whittle, I. R., and Miller, J. D. (1993). “An experimental study of cerebrovascular resistance, pressure transmission and craniospinal compliance," J. Neurosurg. 32, 805-816.

Piper, I. R., Miller, J. D., Dearden, N. M., Leggate, J. R., and Robertson, I. (1990). "Systems analysis of cerebrovascular pressure transmission: An observational study in head-injured patients," J. Neurosurg. 73, 871-880.

Portnoy, H. D., and Chopp, M. (1981). "Cerebrovascular fluid pulse waveform analysis during hypercapnia and hypoxia," J. Neurosurg. 9, 14-27.

Portnoy, H. D., Chopp, M., Branch, C., and Shannon, M. B. (1982). "Cerebrospinal fluid pulse waveform as an indicator of cerebral autoregulation," J. Neurosurg. 56, 668-678.

Reid, A., Marchbanks, R. J., Bateman, D. E., Martin, A. M., Brightwell, A. P., and Pickard, J. D. (1989). "Mean intracranial pressure monitoring by a non-invasive audiological technique: A pilot study," J. Neurol., Neurosurg. Psychiatry 52, 610-612.

Reid, A., Marchbanks, R. J., Burge, D. M., Martin, A. M., Bateman, D. E., Pickard, J. D., and Brightwell, A. P. (1990). "The relationship between in- tracranial pressure and tympanic membrane displacement," Br. J. Audiol. 24, 123-129.

Smelyanskiy, V. N., Luchinsky, D. G., Stefanovska, A., and McClintock, P. V. E. (2005). "Inference of a nonlinear stochastic model of the cardiorespiratory interaction," Phys. Rev. Lett. 94, 098101.

Stettin, E., Paulat, K., Schulz, C., Kunz, U., and Mauer, U. M. (2011). "Noninvasive intracranial pressure measurement using infrasonic emissions from the tympanic membrane," J. Clin. Monit. Comput. 25, 203-210.

Stinson, M. R., and Lawton, B. W. (1989). "Specification of the geometry of the human ear canal for the prediction of sound-pressure level distribution," J. Acoust. Soc. Am. 85, 2492-2503.

Takizawa, H., Gabra-Sanders, T., and Miller, J. D. (1987). "Changes in cerebrospinal fluid pulse wave spectrum associated with raised intracranial pressure," J. Neurosurg. 20, 355-361.

Tavel, M. E. (1978). Clinical Phonocardiography and External Pulse Recording, 3rd ed. (Year Book Medical Publishers Inc., Chicago), pp. 187-220.

Thalen, E., Wit, H., Segenhout, H., and Albers, F. (2002). "Inner ear pressure changes following square wave intracranial or ear canal pressure manipulation in the same guinea pig," Eur. Arch. Otorhinolaryngol. 259, 174-179.

Thalen, E. O., Wit, H. P., Segenhout, J. M., and Albers, F. W. (2004). "Direct measurement flow resistance of cochlear aqueduct in guinea pigs," Acta. Oto-Laryngol. 124(6), 670-674.

Tripathi, K. K. (2004). "Respiration and heart rate variability: A review with special reference to its application in aerospace medicine," Ind. J. Aerospace Med. 81, 64-75.

von Békésy, G. (1936). "On the preparation and measurement of slow sinusoidal variations in air pressure," Ann. Fhysik. 25, 413-432 (in German).

von Békésy, G. (1941). "On the measurement of the amplitude of oscillation of the ossicles by means of the capacitive probe," Akust. Z. 6, 1-16 (in German). [English translation: von Békésy, G. (1960). Experiments in Hearing (McGraw-Hill, New York), pp. 95-104.]

Voss, S. E., Horton, N. J., Tabucchi, T. H., Folowosele, F. O., and Shera, C. A. (2006). "Posture-induced changes in distortion-product otoacoustic emissions and the potential for noninvasive monitoring of changes in intracranial pressure.," Neurocrit. Care. 4(3), 251-257.

Yamaguchi, M., Deguchi, M., Wakasugi, J., Ono, S., Takai, N., Higashi, T., and Mizuno, Y. (2006). "Hand-held monitor of sympathetic nervous system using salivary amylase activity and its validation by driver fatigue assessment," Biosens. Bioelectron. 21, 1007-1014.

Zou, R., Park, E. H., Kelly, E. M., Egnor, M., Wagshul, M. E., and Madsen, J. R. (2008). "Intracranial pressure waves: Characterization of a pulsation absorber with notch filter properties using systems analysis," J. Neurosurg. Pediatrics 2, 83-94.

Zwiener, U., Lüthke, B., Bauer, R., Hoyer, D., Richter, A., and Wagner, H. (1995). "Heart rate fluctuations of lower frequencies than the respiratory rhythm but caused by it," Pflügers Arch. 429, 455-461. 